\title{
Fundamentals of the capacitive resistivity technique
}

\author{
Oliver Kuras, David Beamish, Philip I. Meldrum and Richard D. Ogilvy \\ British Geological Survey, Kingsley Dunham Centre, Keyworth, \\ Nottingham NG12 5GG, United Kingdom
}

(June 17, 2005)

\begin{abstract}
Capacitive resistivity (CR) is an emerging geophysical technique designed to extend the scope of the conventional methodology of DC resistivity to environments where galvanic coupling is notoriously difficult to achieve, for example across engineered structures (roads, pavements), hard rock, dry soil or frozen ground. Conceptually, CR is based on a four-point array capacitively coupled to the ground. Under certain conditions capacitive measurements of resistivity are equivalent to those obtained with the DC technique, thus making DC interpretation schemes applicable to CR data. The coupling properties of practical sensor realizations are shown to be a function of their geometrical arrangement. Separate bodies of theory are associated with two complementary but distinct sensor types: the capacitive line antenna and the plate-wire combination. The use of plate-wire combinations results in localized coupling, which, in conjunction with a quasi-static (low frequency) formulation of the transfer impedance, provides a valid emulation of the DC measurement with point electrodes.

A parametric study of the complex quasi-static transfer impedance reveals the existence of a restricted range of practical parameters that permits successful operation of $\mathrm{CR}$ instruments at low induction numbers. Theory predicts that emulation of the DC measurement is compromised if low-induction-number operation is not maintained throughout a survey area, for example in a zone of high conductivity.
\end{abstract}


Validation of the theory is achieved using specially designed field-scale experiments carried out with a CR instrument capable of measuring the full complex transfer impedance. At intermediate dipole separations, the results are consistent with the predictions of quasi-static theory. Deviations observed in the near and far separation zones can be explained by geometric effects or the breakdown of quasi-static conditions, respectively. Finally, under suitable conditions the phase-sensitive expression for apparent resistivity as a function of the complex transfer impedance is shown to reduce to the classical DC formula for the in-phase component. CR, while capable of emulating DC resistivity, can also be regarded as a physical complement of the inductively coupled ground conductivity method.

\section{INTRODUCTION}

The purpose of the present study is to provide an understanding of the basis for making capacitive resistivity (CR) measurements of the near surface. Electrical resistivity is a fundamental and diagnostic physical property that can be determined using a wide range of geophysical techniques. With the technique described here, the emphasis lies on the capacitive nature of the coupling mechanism between sensors and the ground, in contrast to other resistivity techniques that are either based on galvanic coupling (e.g. DC resistivity) or inductive coupling (electromagnetic techniques). Conceptually, CR draws upon the well-known measurement principle of conventional DC resistivity, which uses a four-point geometric array to determine a transfer resistance, except that galvanic electrodes are replaced by capacitive sensors. From a fundamental standpoint, both techniques share the same generic equivalent electrical circuit model (Wait, 1995). Figure 1 shows the relationship between both techniques. The objectives of the CR technique are (a) to emulate the DC measurement in such a way that popular DC survey techniques (resistivity sounding, profiling, tomographic imaging) can be employed with $\mathrm{CR}$, and (b) to ensure that 
apparent resistivities determined in this fashion comply with established DC interpretation schemes (forward and inverse resistivity modeling).

CR has been developed, both at the research level and commercially, in recent years from existing, founding studies. The earliest successful geophysical application of electrical techniques with capacitive coupling appears to stem from research undertaken in the early 1950s for the U.S. Department of Defense, where the requirement was to detect concealed crevasses in Greenland and Antarctica (Cook, 1956). In the 1970s, pioneering work was carried out in Russia by Timofeev (1973; 1974; 1978). The original Russian application required capacitive coupling to facilitate resistivity measurements on frozen ground for the purpose of mapping permafrost. Later studies, from the 1990s onward, include a variety of empirical, technical and theoretical information that has been incorporated into the current practical designs. Surface measurement systems include the electrostatic quadrupole as described by Grard and Tabbagh (1991), Tabbagh et al. (1993) and Panissod et al. (1998), the multichannel capacitive electrode system as described by Shima et al. $(1995 ; 1996)$ and a number of commercial instruments (Møller, 2001; Lee et al., 2002; Pellerin et al., 2003; Chouteau et al., 2004). Borehole resistivity tools with capacitive sensors have been described by Bristow and Mwenifumbo (1998), Mwenifumbo and Bristow (1999) and Cottineau et al. (2000).

At the outset, the distinction must be made between two different practical realizations of a capacitive sensor, the measurement system and associated theory that ensues. We refer to the first type of system as the capacitive line antenna, and to the second type as the capacitive plate-wire combination. Whilst the former system comprises linear antennas with spatially distributed coupling, the latter system approximates charge-carrying point poles that, in the simplest construction (four poles forming two dipoles), has been termed an electrostatic quadrupole (Grard and Tabbagh, 1991). There are two separate bodies of work dealing with each case. Here, although the physical basis of both types of capacitive measurement system is de- 
scribed, the main emphasis of the study is on the latter type of system due to its closer relationship with conventional DC resistivity.

The bulk resistivity of geological and engineering materials can be more generally described as a complex permittivity that comprises a resistivity component to allow for conduction and a dielectric component to allow for displacement currents. The conventional DC resistivity technique, which requires galvanic contact, can be regarded as a zero or low frequency implementation of electromagnetic (EM) principles of current and voltage association that allows for the investigation of the resistivity component. Very high frequencies, such as those employed by Ground Penetrating Radar (GPR), are associated with electromagnetic wave propagation and enable an assessment of the dielectric component. In an intermediate frequency range, say from $1 \mathrm{kHz}$ to $25 \mathrm{kHz}$, a low-induction-number, quasi-static mode of EM operation exists that is exploited by the capacitive resistivity technique discussed here.

In the CR technique, an oscillating, non-grounded electric dipole is used as a source to generate current flow in the ground. A second dipole of the same type is used to measure the resulting oscillating potential distribution at the ground surface. The dipole-dipole array in its many varieties (Parasnis, 1997) tends to be the most favorable geometry for a capacitive array. The coupling mechanism between the sensors and the ground is predominantly capacitive and inductive effects are, or can be made, negligible. The use of intermediate audio frequencies ensures a quasistatic, low-induction-number mode of operation and may allow the data to simulate the established voltage and current relationships of the conventional DC resistivity method. The degree to which the CR measurement emulates the DC resistivity case, from both a theoretical and empirical viewpoint, is examined in this paper.

We begin by discussing the theoretical framework for the capacitive line antenna and the capacitive plate-wire combination. Conditions that allow a quasi-static mode of operation are established. Subsequently, we investigate the characteristics of realistic capacitive sensors using electrical circuit analysis and compare both sensor types 
on the basis of their respective modeled capacitance. It is shown that the nature of the electrical coupling exerted by the capacitive plate-wire combination implies that this sensor type can act as a point electrode on the scale of the four-point array. Based on these findings, we concentrate on the plate-wire combination and proceed by examining the properties of the complex transfer impedance for point poles as predicted by the electrostatic formalism. The sensitivity of magnitude and phase of the transfer impedance to variations in frequency, geometry and electrical properties is assessed. In the remaining part of this paper, we describe a practical experiment in which $\mathrm{CR}$ measurements were undertaken with a prototype instrument using plate-wire sensors. We discuss the experimental results and assess their compliance with the quasi-static assumption. Finally, we examine how apparent resistivity can be estimated from the measured complex transfer impedance and clarify the relationship between CR and the well-known ground conductivity method.

\section{THEORY}

In this section the existing theory for the two complementary types of capacitive measurement systems is reviewed. The capacitive line antenna, treated as a nongrounded dipole, is considered first. This is followed by a description of the elegant electrostatic formalism for a basic quadrupole, which has been developed to describe localized capacitive sensors. In both cases the context is one of conventional DC resistivity measurements, which provides a description of apparent resistivity in terms of injected current $(I)$ and observed voltage $(\Delta V)$ using a four-electrode arrangement (Figure 2). For a homogeneous halfspace, the apparent resistivity $\left(\rho_{a}\right)$ is equal to the bulk resistivity of the subsurface $(\rho)$ and is constant for any current and electrode geometry:

$$
\rho=\frac{\Delta V}{I} \cdot K^{\mathrm{DC}},
$$

where the term 


$$
K^{\mathrm{DC}}=\frac{2 \pi}{\left[\frac{1}{r_{11}}+\frac{1}{r_{22}}-\frac{1}{r_{12}}-\frac{1}{r_{21}}\right]}
$$

denotes the DC geometric factor. The distance between a current electrode $C_{i}$ and a potential electrode $P_{j}$ is expressed by $r_{i j}$ (Figure 2). On inhomogeneous ground different values of $\rho_{a}$ are obtained if the electrode geometry is altered. Descriptions of geometric factors for a variety of DC resistivity arrays can be found in Telford et al. (1990) or Parasnis (1997).

\section{The capacitive line antenna}

Historically, the practical application of CR and qualitative interpretation of the results appear to have often preceded attempts to develop a more quantitative theoretical framework. An early set of CR instruments, such as the Russian VHCEP system, used various sets of line antennas in the form of cables laid out on the ground. Much of the work is described in an unpublished manuscript by V. M. Timofeev which has been translated to English by G. Rozenberg. This document, edited in Canada by J. A. Hunter, forms the basis of our present understanding of this sensor type. In the original Russian research, line antennas were successfully employed to inject current and measure the electric potential on the ground surface by means of capacitive coupling. However, those measurements could not be interpreted directly by conventional DC resistivity theory because the use of line antennas meant that electrical coupling was no longer restricted to point electrodes. Timofeev therefore developed a modification to the DC geometric factor to account for this obvious violation of the DC concept.

The modified DC geometric factor.-The DC resistivity technique uses grounded dipoles for which the current along the transmitting line (i.e. the connecting wire) and the potential along the receiving line are generally assumed to be constant. The reason is that the conducting wire can be regarded as an equipotential surface for 
static or slowly varying fields, and this remains true even for grounded wires with high contact resistances. However, the assumption breaks down when high-frequency fields are employed, as observed by Timofeev during VLF surveys with grounded dipoles. On a given segment of line antenna close to the surface, the current magnitude was found to decay linearly with distance:

$$
I(x)=\hat{I}\left(1-\frac{x}{l}\right)
$$

where $\hat{I}$ is the input current, $l$ the length of the antenna segment and $x$ the distance along the segment. We will show at a later stage that this empirical observation can be understood by examining the equivalent electrical circuit of a capacitive line antenna.

Based on the current distribution (3), Timofeev showed that a nominal DC geometric factor can be calculated for the line antenna arrangement by discretizing it into a series of four-point arrays, each associated with a corresponding portion of the total current. For the inline dipole-dipole array this factor is given by the integral (Lee et al., 2002)

$$
\frac{1}{K^{\mathrm{LA}}}=\frac{\Delta V}{\rho I}=\frac{1}{\pi} \int_{0}^{l_{T}} \int_{0}^{l_{R}} \frac{I\left(x_{1}\right)}{\hat{I}} \cdot \frac{I\left(x_{2}\right)}{\hat{I}} \cdot \frac{1}{\left(r-x_{1}+x_{2}\right)^{3}} d x_{1} d x_{2},
$$

where $l_{T}$ and $l_{R}$ denote the lengths of the transmitter and receiver antennas, respectively, and $r$ the separation between them. The parameters $x_{1}$ and $x_{2}$ are distance coordinates along the transmitter and receiver antennas, respectively. For a symmetric inline dipole-dipole array $\left(l_{T}=l_{R}\right)$, the integral expression can be written as (Lee et al., 2002)

$$
K^{\mathrm{LA}}=\frac{\pi l}{\ln \left(\left(\frac{b^{2}}{b^{2}-1}\right)^{2 b}\left(\frac{b^{2}+2 b}{(b+1)^{2}}\right)^{b+2}\left(\frac{b^{2}-2 b}{(b-1)^{2}}\right)^{b-2}\right)},
$$

where

$$
b=\frac{2 r}{l} .
$$


With a known current injected at the feeding point of one antenna and the potential measured at the feeding point of the second antenna, this method can be used to calculate an apparent resistivity of the subsurface. However, it should be noted that the entire length of the antenna contributes to the measurement, both in terms of injected current and observed potential. We will show in the following that this may have important consequences for the quantitative interpretation of resistivity data obtained with capacitive line antennas.

Concept of the equivalent grounded dipole.-Suppose capacitive line antennas are employed to form a symmetric dipole-dipole array and apparent resistivity measurements are conducted over a homogeneous subsurface. An array of conventional grounded dipoles that measures the same transfer impedance as the line antenna array can then be regarded as the equivalent grounded array (Figure 3). As the apparent resistivity over a homogeneous subsurface is independent of array geometry, the geometric factor $K^{\mathrm{DC}}$ of such an equivalent grounded array must satisfy the condition $K^{\mathrm{DC}}=K^{\mathrm{LA}}$. Consequently, given a dipole separation $r$, the length of the equivalent grounded dipoles $l_{\mathrm{DC}}$ will generally be different from the length of the line antenna dipoles $l_{\mathrm{LA}}$ for the geometric factors to be equal. This is important if forward and inverse modeling algorithms developed for DC resistivity (and grounded dipoles with point electrodes) are to be applied to CR data obtained with capacitive line antennas. In order to calculate the length of the equivalent grounded dipole $l_{\mathrm{DC}}$ explicitly, Timofeev's geometric factor (5) must equal the DC geometric factor of the conventional symmetric inline dipole-dipole array with dipole separation $r_{\mathrm{DC}}$ and dipole length $l_{\mathrm{DC}}$ (Parasnis, 1997):

$$
K_{\mathrm{DDin}}^{\mathrm{DC}}=\frac{2 \pi}{\frac{2}{r_{\mathrm{DC}}}-\frac{1}{r_{\mathrm{DC}}-l_{\mathrm{DC}}}-\frac{1}{r_{\mathrm{DC}}+l_{\mathrm{DC}}}}=\pi r_{\mathrm{DC}}\left(1-\frac{r_{\mathrm{DC}}^{2}}{l_{\mathrm{DC}}^{2}}\right),
$$

where $r_{\mathrm{DC}}>l_{\mathrm{DC}}$. Numerical comparison of expressions (5) and (7) shows that the capacitive line antenna dipole is always longer than its grounded equivalent. For large separations $r$, the length of the equivalent grounded dipole is approximately 
half the length of the line antenna $\left(l_{\mathrm{DC}} / l_{\mathrm{LA}} \approx 0.5\right)$. For $r>4 l$ the deviation from this intuitive value is smaller than $1 \%$. At short separations however, the equivalent DC dipole can be significantly longer than $0.5 l_{\mathrm{LA}}$. For $r \leq 1.5 l$, which is not an unusual configuration for line antenna systems, one obtains $l_{\mathrm{DC}} / l_{\mathrm{LA}} \geq 0.535$, i.e. the deviation is already greater than $7 \%$. As a typical example, consider a line antenna dipole-dipole array with $l_{\mathrm{LA}}=10 \mathrm{~m}$ and $r_{\mathrm{LA}}=15 \mathrm{~m}$. The spacing between the inner dipole endpoints for such an array would be $5 \mathrm{~m}$ (Figure 3). Intuitively, one might expect the equivalent grounded array to be $l_{\mathrm{DC}}=5 \mathrm{~m}$ and $r_{\mathrm{DC}}=15 \mathrm{~m}$. However, the DC geometric factor of such an array $\left(K^{\mathrm{DC}} \approx 376.9\right)$ would exceed the geometric factor of the line antenna array ( $K^{\mathrm{LA}} \approx 326.2$ ) by nearly $16 \%$, resulting in a significant overestimation of apparent resistivity. It is therefore advisable to explicitly calculate the equivalent dipole length if line antenna data are to be interpreted quantitatively by means of conventional DC forward or inverse modeling.

\section{The electrostatic quadrupole}

When localized capacitive sensors were first used in the early 1990s, a new theoretical framework was required to describe their specific properties. The concept of an "electrostatic quadrupole", introduced by Grard (1990), was the key idea for the perception of $\mathrm{CR}$ as a generalization of DC resistivity. Rather than restricting the theoretical formulation to a particular sensor design with finite surface extent, Grard's concept uses point poles to represent the electrodes. This section discusses details of this concept and redevelops Grard's "electrostatic formalism" with a simplified and updated notation.

Electrostatic charge near an air-earth interface.-The electrostatic formalism assumes electrostatic point charges near a planar interface between two homogeneous and isotropic halfspaces representing electrically different media (Grard, 1990). A charge located in the vicinity of the interface will create an electrostatic potential in 
its surroundings that is a function of the dielectric properties of both media and can be readily calculated using the theory of images (Figure 4). Suppose a charge $Q$ is located at $C$ and its image charge $Q^{\prime}=-Q$ at $C^{\prime}$, then the potential at a point $P$ is given by

$$
V(P)=\frac{Q}{4 \pi \varepsilon^{(I)}}\left(\frac{1}{r}-\alpha \frac{1}{r^{\prime}}\right)
$$

where $r$ corresponds to the distance $\overline{C P}$ and $r^{\prime}$ to the distance $\overline{C^{\prime} P}$. Compared with the free space expression, equation (8) contains an additional contribution due to the image charge. The real factor $\alpha$ comprises the dielectric permittivities of the two media:

$$
\alpha=\frac{\varepsilon^{(I I)}-\varepsilon^{(I)}}{\varepsilon^{(I I)}+\varepsilon^{(I)}} .
$$

In the case of an air-earth interface, these are given by

$$
\begin{gathered}
\varepsilon^{(I)}=\varepsilon_{0} \\
\varepsilon^{(I I)}=\varepsilon_{0} \varepsilon_{r} .
\end{gathered}
$$

For static charges, $\alpha$ is therefore fully determined by the relative permittivity (dielectric constant) of the subsurface:

$$
\alpha=\frac{\varepsilon_{r}-1}{\varepsilon_{r}+1} .
$$

The quadrupole concept.- - It is now straightforward to extend this result to a four-pole arrangement. A practical electric current source has two poles carrying opposite charges at any time. Equally, a practical measurement of the electric potential can only be carried out with respect to a reference. This leads to the concept of an electrostatic quadrupole where two poles $C_{1}, C_{2}$ carrying charges $+Q$ and $-Q$, respectively, act as a current source, while the potential difference $\Delta V=V_{1}-V_{2}$ is measured between the two remaining poles $P_{1}, P_{2}$ (Figure 5). This potential difference is then given by (Grard, 1990) 


$$
\Delta V=\frac{Q}{4 \pi \varepsilon_{0}}\left(\frac{1}{r_{11}}+\frac{1}{r_{22}}-\frac{1}{r_{12}}-\frac{1}{r_{21}}-\alpha\left(\frac{1}{r_{11}^{\prime}}+\frac{1}{r_{22}^{\prime}}-\frac{1}{r_{12}^{\prime}}-\frac{1}{r_{21}^{\prime}}\right)\right) .
$$

This expression can be simplified by introducing the electrostatic geometric factor

$$
K^{\mathrm{ES}}=\frac{\frac{1}{r_{11}^{\prime}}+\frac{1}{r_{22}^{\prime}}-\frac{1}{r_{12}^{\prime}}-\frac{1}{r_{21}^{\prime}}}{\frac{1}{r_{11}}+\frac{1}{r_{22}}-\frac{1}{r_{12}}-\frac{1}{r_{21}}}
$$

which contains the distances between all poles, including the virtual poles carrying image charges. It is also useful to recognize that

$$
C_{0}=\frac{4 \pi \varepsilon_{0}}{\frac{1}{r_{11}}+\frac{1}{r_{22}}-\frac{1}{r_{12}}-\frac{1}{r_{21}}}
$$

has the dimensions of a capacitance and, although this is purely formal, can be regarded as a mutual capacitance of the configuration in free space. The potential difference can then be expressed as

$$
\Delta V=\frac{Q}{C_{0}}\left(1-K^{\mathrm{ES}} \alpha\right)
$$

For a given pair of static charges in the vicinity of a homogeneous earth, the voltage between any two neighboring points can thus be calculated if all four positions and the dielectric properties are known.

The quadrupole in quasi-static approximation.-The formulation so far has been entirely based on electrostatics, i.e. the results are strictly valid only for charges that are constant over time. For charges varying slowly with time, $Q=Q(t)$, equation (15) can be used to derive a simple time-dependent expression for the potential. Suppose an alternating current $I=I(t)$ is injected across $C_{1}$ and $C_{2}$, then instantaneous charges $Q_{1}=-Q_{2}$ with a magnitude of $\left|Q_{1}\right|=\left|Q_{2}\right|=Q$ will be carried by the two poles. These charges can be expressed as the time integral of the injected current and with $I=\dot{Q}$ one obtains for harmonic currents

$$
Q(t)=\int_{t_{0}}^{t} I(\tilde{t}) d \tilde{t}=\frac{1}{i \omega} I(t)+\text { const. }
$$

The integration constant represents an arbitrary static charge at time $t_{0}$ that has no effect on the time-varying process and can be neglected. Insertion into equation (15) makes the expression for the potential difference time-dependent: 


$$
\Delta V(t)=\frac{I(t)}{i \omega C_{0}}\left(1-K^{\mathrm{ES}} \alpha\right)
$$

As charge, current and potential become a function of time, the dielectric permittivities in equation (9) must now be regarded as complex quantities. As the injection of current across $C_{1}$ and $C_{2}$ implies electric current flow in the ground, the observed potential will be a function of subsurface resistivity. The general form of the complex permittivity is

$$
\varepsilon=\varepsilon_{0} \varepsilon_{r}-i \frac{1}{\rho \omega}
$$

i.e. it contains resistivity in its imaginary component and relative permittivity in its real component. In the case of the air-earth interface, the relevant complex permittivities are

$$
\begin{aligned}
\varepsilon^{(I)} & =\varepsilon_{0} \\
\varepsilon^{(I I)} & =\varepsilon_{0} \varepsilon_{r}-i \frac{1}{\rho \omega} .
\end{aligned}
$$

Accordingly, the factor $\alpha$ (equation 11) is now also a complex number:

$$
\alpha=\frac{\rho \omega \varepsilon_{0}\left(\varepsilon_{r}-1\right)-i}{\rho \omega \varepsilon_{0}\left(\varepsilon_{r}+1\right)-i} .
$$

It comprises the electrical properties of both media together with the frequency of operation and is representative of the electrical behavior of the arrangement.

The transfer impedance

$$
Z=\frac{\Delta V}{I}
$$

between the current dipole and the potential dipole can be regarded as the characteristic response function of the quadrupole. In equation (17), it is useful to identify

$$
Z_{0}=\frac{1}{i \omega C_{0}}
$$

as the transfer impedance of the quadrupole in free space $(\alpha=0)$. The result is a simple relation for the complex transfer impedance: 


$$
Z=Z_{0}\left(1-K^{\mathrm{ES}} \alpha\right)
$$

The deviation from the free space value $Z_{0}$ is given by the product of the geometric factor $K^{\mathrm{ES}}$ and the complex factor $\alpha$ representing the electrical properties of the subsurface.

\section{The quasi-static regime}

The above equations have been derived by formally inserting a time-varying charge function associated with a time-varying current $I(t)$. The procedure ignores the electrodynamic framework of Maxwell's equations in which $I(t)$ is associated with an oscillating electromagnetic field and associated induced secondary currents. Therefore, conditions needed to be established under which such electromagnetic effects could be neglected for practical quadrupoles. Grard and Tabbagh (1991) argue that the quasi-static approximation applies and equation (17) remains valid if the wavelengths $\lambda^{(I)}, \lambda^{(I I)}$ of an electromagnetic wave of angular frequency $\omega$ in media of permittivities $\varepsilon^{(I)}, \varepsilon^{(I I)}$ are much greater than the characteristic distances $r$ and $r^{\prime}$ in the respective medium. In electromagnetic terms, this corresponds to the well-known condition of a low-induction-number regime, i.e. the characteristic distances involved must be small compared with the electromagnetic skin depth.

The induction number $B$ is a means of describing the nature of EM induction as a function of geometry and electrical properties. $B$ is defined as the ratio between a characteristic measurement scale length $L$ and the electromagnetic skin depth $\delta$ $\left(\mathrm{McNeill,}\right.$ 1980) ${ }^{1}$ :

$$
B=\frac{L}{\delta}=L \sqrt{\frac{\omega \mu_{0} \sigma}{2}}
$$

\footnotetext{
${ }^{1}$ Sometimes the induction number is defined as the square of expression (24), neglecting the constant factor $1 / 2: B^{\prime}=L^{2} \omega \mu_{0} \sigma$.
} 
A broad class of applied electromagnetic instruments employ an equivalent lowinduction-number mode of operation. Based on experience from applying the electrostatic technique to long-offset resistivity sounding, Benderitter et al. (1994) proposed the condition:

$$
B^{2} \ll 1
$$

If this condition is violated, equations (17) and (23) are no longer valid as the quasistatic approximation breaks down and inductive effects become significant. The characteristic scale length $L$ is taken to be the transmitter-receiver separation $r$. Equation (25) then provides an upper frequency limit for quasi-static operation:

$$
f<f_{U}=\frac{\rho}{\pi \mu_{0} r^{2}}
$$

The limiting frequency $f_{U}$ decreases with separation as $1 / r^{2}$ and is proportional to resistivity; in other words, the condition can be fulfilled with either larger separations in more resistive environments or shorter separations in more conductive environments. If we assume $r<10 \mathrm{~m}$ for near surface applications and a lower resistivity limit of $10 \Omega \mathrm{m}$, an upper frequency limit of approximately $25 \mathrm{kHz}$ is obtained. The low-induction-number condition (25) simultaneously satisfies the wavelength conditions defined earlier: in the above example $(25 \mathrm{kHz})$ the free space wavelength is approximately $12,000 \mathrm{~m}$, whereas in a $10 \Omega \mathrm{m}$ medium the wavelength reduces to $45 \mathrm{~m}$.

In summary, frequency of operation is the fundamental parameter that determines whether inductive effects are significant or can be neglected. This condition is restrictive in terms of an upper frequency limit. On the other hand, frequency is also significant for the capacitive coupling mechanism as the transmitter is required to inject an electric current across a dipole representing a predominantly capacitive load. We will now show that this condition is restrictive in terms of a lower frequency limit. 


\section{CAPACITIVE SENSORS}

Thus far, electric current has been treated as an independent parameter with constant magnitude. However, in a practical instrument the output current is likely to be a function of a variety of external factors, including sensor design. A simple electric circuit model can be used to estimate the current in the transmitter output circuit for a given frequency. The transmitter circuit may be modeled as the ground resistance in series with the capacitance of the sensors, driven by the electromotive force of the generator (Shima et al., 1995). This corresponds to the outer circuit on the right-hand side of Figure 1. The load represents a complex impedance of

$$
Z(\omega)=X_{C}+R=\frac{1}{i \omega C}+R
$$

where $R$ represents the resistive, $X_{C}$ the reactive component of the impedance and $C$ the effective sensor capacitance. It is worth noting that $R$ mainly comprises the ohmic ground resistance as seen by the transmitter circuit. This is in contrast to the transfer resistance typically measured in DC resistivity, which is calculated from the injected current and the voltage measured at the receiver. Here, the voltage $U_{\text {Tx }}$ generated by the transmitter (or $E$ in the notation of Shima et al.) is relevant. Its application at the terminals will result in a current flow

$$
I=\frac{U_{\mathrm{Tx}}}{Z(\omega)}
$$

A practical aim both in DC resistivity and CR is to create high electric dipole moments at the transmitter so that the observed signal at the receiver is above detection level for a given array separation. This is particularly important for electrode arrays with large geometric factors. From experience with DC resistivity, currents of the order of $10 \mathrm{~mA}$ and higher are typically required for shallow investigations. To achieve this, the complex load at the transmitter output must be overcome. For example, a portable battery-powered transmitter with an output power of $10 \mathrm{~W}$ can drive a $10 \mathrm{~mA}$ current 
at voltages of $1000 \mathrm{~V}$. The maximum output impedance this transmitter would be able to overcome while maintaining a constant current would be of the order of $10^{5} \Omega$.

Realistic values for the sensor capacitance are crucial for a meaningful estimate of the total impedance. Shima et al. (1995) have used the parallel-plate approximation to estimate sensor capacitance. Whilst this may suffice for an approximate calculation, a more detailed analysis is required if the influence of sensor geometry is of interest. The parallel-plate formula states

$$
C=\varepsilon_{r} \varepsilon_{0} \frac{A}{d}
$$

where $A$ is the area of the plates and $d$ their separation. The factor $A / d$ will be typically of the order of $10^{2} \mathrm{~m}$ so that capacitances in air $\left(\varepsilon_{r}=1\right)$ are expected to be of the order of $C \approx 1 \mathrm{nF}$. It is safe to assume that the ohmic ground resistance (typically a few $\Omega$ ) is negligible with respect to the sensor reactance, so that the total impedance becomes

$$
Z(\omega) \approx \frac{1}{i \omega C}
$$

We have verified this assumption through practical measurements. Hence, to keep the impedance below a desired threshold, the frequency must satisfy

$$
f \geq f_{L}=\frac{I}{2 \pi C U_{\mathrm{Tx}}} .
$$

For the above values, i.e. a typical capacitance of $1 \mathrm{nF}$ and an impedance limit of $10^{5} \Omega$, one obtains a lower frequency limit of $f_{L}=1.6 \mathrm{kHz}$.

\section{Capacitance of realistic sensors}

The above analysis shows that CR measurements are critically dependent on sensor capacitance. The capacitance of the transmitter dipole controls current injection into the ground. Larger capacitance is associated with a lower output impedance so that higher currents can be generated. The passive receiver dipole measures the 
electric field immediately above the ground surface due to the current flow generated by the transmitter. For both types of dipole, the sensor capacitance is a quantitative measure for the degree of electrical coupling with the ground.

For realistic sensors, the parallel plate model is oversimplistic because it assumes plates of infinite area. A more accurate estimate of sensor capacitance can be obtained by modeling the capacitance of geometric structures. The capacitance of a conductor over a ground plane is determined by the geometric shape of the conductor, its perimeter and the distance of the conductor from the surface. Essentially, the relation

$$
C=\frac{Q}{U}=\frac{1}{U} \iint \sigma d S
$$

is used to calculate capacitance, where $S$ is the surface of the conductor and $\sigma$ is the surface charge density. When a voltage $U$ with respect to ground is applied, surface charges occur on both the ground plane and the conductor. Their density $\sigma$ is a measure of the discontinuity of the normal component $D_{n}$ of the dielectric flux density $\mathbf{D}=-\varepsilon_{0} \varepsilon_{r} \nabla V$ :

$$
D_{n_{1}}-D_{n_{2}}=\sigma
$$

The potential $V$ can be calculated from Laplace's equation by solving the associated boundary-value problem (Ringhandt and Wagemann, 1993). Here, the relevant geometries are those of a wire segment and a thin plate above a ground plane.

Wire segment above a ground plane.-An infinite cylindrical conductor suspended over the ground plane represents a two-dimensional problem for which an analytical solution to equation (32) exists. The capacitance per unit length of such a conductor is given by (Smythe, 1950; Yuan and Trick, 1982)

$$
\hat{C}=\frac{2 \pi \varepsilon_{0} \varepsilon_{r}}{\ln \left(\frac{d+\sqrt{d^{2}-a^{2}}}{a}\right)}=\frac{2 \pi \varepsilon_{0} \varepsilon_{r}}{\operatorname{acosh}\left(\frac{\mathrm{d}}{\mathrm{a}}\right)},
$$

where $a$ is the radius of the conductor and $d$ is the distance from its center to the ground plane. 
Thin plate above a ground plane.-The capacitance of a rectangular plate of finite thickness suspended over the ground plane has no analytical solution. An approximate functional expression is given by Sakurai and Tamaru (1983) as:

$$
C=\varepsilon_{0} \varepsilon_{r} \cdot\left[1.15 \frac{w \cdot l}{h}+1.40\left(\frac{t}{h}\right)^{0.222} \cdot(2 w+2 l)+4.12\left(\frac{t}{h}\right)^{0.728} \cdot h\right] .
$$

Here, $w$ and $l$ are the width and length of the plate, respectively, $t$ its thickness and $h$ the elevation of the bottom face above the ground surface. Compared with the parallel-plate approximation (29), the capacitance of a single plate is greater because the opposing "plate" of the capacitor is now replaced by the infinitely wide ground plane, and fringing fields at the edges and corners of the plate must be taken into account.

\section{Implications for the capacitive line antenna}

We have shown earlier that it is likely that a total sensor capacitance of the order of $1 \mathrm{nF}$ will be required in order to limit the transmitter output impedance to practical values at audio frequencies. Capacitances much lower than $100 \mathrm{pF}$ may be inadequate to provide sufficient capacitive coupling. According to the conceptual model of a line antenna (Figure 6), the antenna can be regarded as a composite of wire segments of equal length, each having a distinct capacitance. Following equation (34), a wire segment with $d / a=6$ (e.g. $d=6 \mathrm{~mm}, a=1 \mathrm{~mm}$, corresponding to a wire of $2 \mathrm{~mm}$ diameter elevated above ground with a $5 \mathrm{~mm}$ air gap) has a capacitance per unit length of $\hat{C}=22.5 \mathrm{pF} / \mathrm{m}$. By analogy with the parallel-plate capacitor, greater capacitances are observed if the wire is closer to the surface, i.e. for smaller values of $d / a$. However, note that ratios much smaller than $d / a=2$ are unrealistic due to the finite extent of the sheathing required for electrical insulation of practical wires. The results demonstrate that suitable conditions exist under which line antennas may be employed successfully, however a minimum length condition applies. For relevant 
ratios of $d / a$, total capacitances of the order of $1 \mathrm{nF}$ are reached only by line antennas with a length of several meters, particularly for $d / a \geq 2$.

This conceptual model can also help explain the linear decrease in electrical current along the antenna as observed by Timofeev. The simplified equivalent circuit model of a line antenna comprises $n$ identical capacitors connected in parallel (Figure 6). Hence the total capacitance of a symmetric non-grounded dipole is given by the sum of the capacitances of the individual segments:

$$
C_{\mathrm{LA}}=\frac{1}{2} \sum_{i=1}^{n} C_{i}
$$

The division by two is due to the two poles connected in series with each other. In this model, an identical amount of current $I_{i}$ leaks to the ground through each individual capacitive segment $C_{i}$ :

$$
I_{i}=\frac{U}{Z_{i}}=i \omega U C_{i}
$$

where $U$ is the voltage across the antenna, which is assumed to be constant in this model as the antenna itself represents an equipotential surface. The electrical current observed along the antenna can thus be described by equation (3), as current leakage is proportional to distance.

\section{The plate-wire combination}

The conceptual model of a plate-wire combination is shown in Figure 7. Its total sensor capacitance is given by the sum of the individual components $\left(C_{\text {Plate }}\right.$ and $\left.C_{\text {Wire }}\right)$, which act as parallel capacitors. A practical dipole consists of two plate-wire components in series so that the total dipole capacitance is simply

$$
C_{\text {PWDipole }}=\frac{1}{2}\left(C_{\text {Plate }}+C_{\text {Wire }}\right) .
$$

The key objective in designing a plate-wire sensor is to make $C_{\text {Wire }}$ negligible with respect to $C_{\text {Plate }}$. This condition is intended to make the sensor act in a "polelike" manner, so that coupling will be focussed on a small area and the electrostatic 
formalism can be applied. The objective is most simply achieved by using a minimum elevation for the plate and ensuring that the wire is sufficiently raised above the ground surface. However, using equations (35) and (34) it can be shown that, for sufficiently small elevations (e.g. approaching $1 \mathrm{~mm}$ ), $C_{\text {Wire }}$ increases dramatically and exceeds $C_{\text {Plate }}$ (Kuras, 2002). The sensor design must aim to avoid this situation, as the plate would serve no purpose and the majority of the current would be injected through the wire segment. As elevation increases to, say, several millimeters, the capacitance of the wire segment falls off rapidly to well below the capacitance of the plate, which decreases more slowly.

\section{PROPERTIES OF THE COMPLEX TRANSFER IMPEDANCE}

The above results give rise to the hypothesis that a CR measurement with capacitive plate-wire sensors may be adequately described by the electrostatic formalism. The experimental validation of this fundamental concept with a practical CR instrument is the goal of this study. To understand the general properties of the quadrupole in quasi-static approximation, it is useful to examine the functional behavior of the complex transfer impedance over a wide range of parameters. For this purpose, Grard (1990) considered the normalized transfer impedance

$$
\frac{Z}{\left|Z_{0}\right|}=-i\left(1-K^{\mathrm{ES}} \alpha\right)
$$

whose modulus varies in the range between 0 and 1 , while the phase relation and algebraic sign of $Z$ are retained. A plot of magnitude and phase of $Z /\left|Z_{0}\right|$ as a function of the generalized frequency

$$
\Omega=\rho \omega \varepsilon_{0}\left(\varepsilon_{r}+1\right)
$$

is shown in Figure 8. Using this generalized frequency, Grard was able to show that three different characteristic regimes can be distinguished for $Z /\left|Z_{0}\right|$, depending on 
the electromagnetic balance between the dielectric term $\varepsilon_{0} \varepsilon_{r}$ and the resistivity term $(\rho \omega)^{-1}$ in the expression for complex permittivity (18). All relevant parameters that determine this balance (frequency, resistivity and relative permittivity) are embedded in $\Omega$. Analysis of some numerical examples (Table 1) reveals that under the circumstances likely to be encountered in CR one can expect $\Omega \ll 1$. However, the size of $\Omega$ is not necessarily an indicator for quasi-static conditions. In the numerical example, all three regimes of $\Omega$ are associated with low induction numbers (Table 1) and thus quasi-static conditions. Instead, $\Omega \ll 1$ is equivalent to a situation in which the resistivity term dominates and conduction currents outweigh displacement currents. Under such conditions the interaction of the electromagnetic field with the earth is governed by diffusion rather than wave propagation. For $\Omega \ll 1$ and $K^{\mathrm{ES}} \approx 1$ (the typical conditions for $\mathrm{CR}$ ), the magnitude of the transfer impedance will be small with respect to its free space value (Figure 8a) and the observed potential is likely to be in phase with the injected current, although strong phase shifts are not impossible - particularly for $K^{\mathrm{ES}}<1-10^{-5}$ (Figure $8 \mathrm{~b}$ ).

\section{Parameter study}

In addition to this general assessment, we have undertaken a detailed parametric study in which the functional dependence of the complex transfer impedance was examined with respect to individual parameters (frequency, array geometry, electrical properties). The aim was to determine whether a realistic range of parameters exists that could be implemented in a practical CR instrument and would allow successful operation of the CR technique under quasi-static conditions.

\section{Variation with frequency}

Restrictions on the choice of frequency have already been discussed in the context of the quasi-static approximation and sensor capacitance. We have demonstrated that 
the range of frequencies of operation is constrained by an upper limiting frequency $(25 \mathrm{kHz})$ associated with the condition of low induction numbers and a lower limiting frequency $(1.6 \mathrm{kHz})$ as a result of the need to inject sufficiently high currents via the transmitter dipole. These limitations apply under all circumstances. However, even in quasi-static approximation, the transfer impedance $Z$ remains a function of frequency. As an example, the electrostatic potentials observed with a square electrostatic array $(r=1 \mathrm{~m})$ at an elevation of $1 \mathrm{~mm}$ are shown in Figure 9 . The calculation is based on equation (23), assuming a constant current of $10 \mathrm{~mA}$. Relative permittivity was set to a typical value of $\varepsilon_{r}=10$, as a range of $3 \leq \varepsilon_{r} \leq 40$ is likely to cover most practical ground conditions (e.g. Topp et al., 1980). Curves for a range of resistivities from 0.1 to $1000 \Omega \mathrm{m}$ are displayed. The frequencies considered span four orders of magnitude and extend beyond the limiting frequencies $f_{L}$ and $f_{U}$. The values of an equivalent DC array are shown as dashed lines. Figure 9a shows that, in the frequency range relevant for CR, the magnitude of the potential can be expected to vary strongly with frequency in conductive environments, i.e. for resistivities lower than $10 \Omega \mathrm{m}$. This is in contrast to the DC potentials, which are frequency-invariant. Above a certain frequency specific to the value of resistivity, the CR magnitudes assume a constant level and cease to be sensitive to frequency variation. The threshold frequencies are higher for more conductive ground.

Phase angles occur only in the CR potentials (Figure 9b). It should be noted that in equation (23) the phase is negative throughout, i.e. the response represents a phase lag, as expected for a capacitive impedance. The plots show that for the relevant frequency range, significant phase lags can be expected for conductive ground, while higher resistivities are more likely to be associated with an in-phase response.

The results demonstrate that there is no one single "ideal" frequency of operation in $\mathrm{CR}$ and any practical choice of frequency will represent a trade-off between the contradicting requirements described above. Values sufficiently distant from the two limiting frequencies $f_{L}$ and $f_{U}$ are likely to be a good compromise. We therefore 
conduct all further calculations in this study at a fixed frequency of $15 \mathrm{kHz}$.

\section{Variation with array geometry}

Dimensions.-By analogy with the DC case, the CR transfer impedance is a function of array dimensions. The limiting condition for the choice of array style in CR is the influence of the connecting wires on sensor capacitance. The most appropriate array style for use with capacitive sensors is therefore the dipole-dipole array, which allows feed lines to have minimal length. Amongst the variety of conceivable dipoledipole geometries, the equatorial type (Parasnis, 1997) has a number of advantages, including compact size and superior depth of investigation relative to overall array dimensions. For the sake of clarity, we therefore present results for the equatorial dipole-dipole array only. The inline array is discussed in Kuras (2002). Due to scalability it is sufficient to examine unit dipoles $(l=1 \mathrm{~m})$ at varying separations.

Figure 10 shows the variation of the complex potential in magnitude and phase with dipole separation $r$, ranging from $0.1 \mathrm{~m}$ to $100 \mathrm{~m}$. The length of the dipoles is $1 \mathrm{~m}$. Array elevation was assumed constant at $1 \mathrm{~mm}$. Corresponding real-valued potentials for an equivalent DC array are shown as dashed lines. Curves have been plotted for a range of resistivities between $10^{-1}$ and $10^{3} \Omega \mathrm{m}$. In accordance with expectations, the observed voltage is proportional to resistivity, so that higher magnitudes are observed in resistive environments, while a conductive subsurface results in significantly lower voltages (Figure 10a). For large separations, the CR magnitude is seen to vary as $1 / r^{2}$, in accordance with what would be expected for DC dipoles. This behavior is more obvious in resistive environments. For low resistivities and small separations however, a distinct deviation from the corresponding DC curves can be observed. Under such conditions, the magnitude of the CR potential is higher than the corresponding DC potential due to the influence of the quadrature component. By setting a minimum voltage above which signal detection is considered realistic, a maximum separation for 
reliable operation can be derived directly from the graph. For example, assuming that voltages of the order of $10^{-6} \mathrm{~V}$ can still be detected under favorable signal-to-noise conditions, the maximum useful dipole separation in a $10 \Omega \mathrm{m}$ environment would be approximately $25 \mathrm{~m}$.

The sensitivity to phase is apparent from Figure 10b. In resistive environments, the potentials are nearly always in phase, regardless of separation. In conductive environments, in-phase behavior is still observed at larger distances, but significant phase shifts may occur at small separations, where the response tends towards quadrature $(-\pi / 2)$, i.e. free space conditions. For a given separation, the phase shift can increase dramatically as resistivity decreases. For example, with a rectangular array with $r=2 \mathrm{~m}$ one would observe phase angles of $-2.47 \mathrm{mrad}$ at $1000 \Omega \mathrm{m},-8.17 \mathrm{mrad}$ at $100 \Omega \mathrm{m},-79.9 \mathrm{mrad}$ at $10 \Omega \mathrm{m},-675 \mathrm{mrad}$ at $1 \Omega \mathrm{m}$, and $-1450 \mathrm{mrad}$ at $0.1 \Omega \mathrm{m}$.

We have found the response for the equatorial configuration to be very similar to that of the inline dipole-dipole array, except the magnitude of the potential of the equatorial array is smaller. This is consistent with the fact that the main symmetry axis of the equatorial configuration is perpendicular to the main symmetry axes of the individual dipoles, causing the "coupling" between potential and current dipole to be weaker.

Elevation.-In contrast to the DC case, elevation of the capacitive array above the ground surface also has an influence on the transfer impedance. The size of this effect is important for the design of practical CR instruments, because it is unlikely that the effective elevation of a real capacitive sensor over the ground surface can be determined with sufficient accuracy during a practical measurement. For the purposes of this study, we consider four electrodes having the same elevation, i.e. the entire array is assumed to be at a constant height above ground. This assumption does not represent a serious restriction because the geometric factor, and hence the transfer impedance, does not change fundamentally if different electrodes have slightly 
different elevations, as would be expected for measurements on natural surfaces and across uneven terrain. Again, we consider unit dipoles due to scalability, this time at a fixed separation of $1 \mathrm{~m}$ (i.e. a unit square array).

Figure 11 shows the variation of the complex potential in magnitude and phase with array elevation. The corresponding DC potentials for an equivalent grounded array are shown as dashed lines. As before, curves have been plotted for five different resistivities. The range of elevations considered is between $10^{-4}$ and $10^{-1} \mathrm{~m}$, corresponding to capacitive sensors lying on, or close to, the ground.

As with the DC case, the magnitude of the observed CR potential at very small elevations is constant, and only a function of resistivity (Figure 11a). This is an important observation because it means that small changes in elevation, which may be difficult to account for in a practical field setup, are unlikely to have a significant effect on the measurement. At larger elevations however, magnitudes start to increase and eventually show asymptotic behavior towards the free space value. The point where this occurs is a function of resistivity. In free space the transfer impedance is not sensitive to resistivity. Large sensor elevations must therefore be avoided. Note that throughout this parameter study the injected current is assumed constant, but in practice an increase in sensor elevation is associated with an increase in impedance in the transmitter output circuit. Realistically, the current (and hence the observed potential) must therefore decrease, unless additional power is provided by the generator.

The CR phase curves show a fundamental change associated with the transition from the DC case (zero elevation) to free space conditions (Figure 11b). At small elevations the observed voltage is largely in phase, in accord with the DC case. At larger elevations the quadrature component increases and a phase rotation towards $-\pi / 2$ is observed. This corresponds to free space conditions where the CR transfer impedance is entirely in quadrature. 


\section{Variation with electrical properties}

The electrical properties of the subsurface (resistivity and relative permittivity) are the only practical variables in a CR measurement and any variation in the measured transfer impedance will be attributed to a variation in electrical properties.

Resistivity.- The variation with resistivity of the complex potential in magnitude and phase for an equatorial dipole-dipole array is shown in Figure 12. Dashed lines represent the corresponding potentials for an equivalent DC array. Curves have been plotted for five different dipole separations likely to be relevant in near-surface investigations, namely from $1 \mathrm{~m}$ (i.e. a unit square array) to $25 \mathrm{~m}$. The array elevation is fixed at $1 \mathrm{~mm}$. The range of resistivities considered is between $10^{-1}$ and $10^{3} \Omega \mathrm{m}$.

The magnitude of the CR potential is found to match the DC curves almost everywhere except at low resistivities and small dipole separations $(<1 \mathrm{~m})$, where CR magnitudes remain at constant levels despite a further decrease in resistivity (Figure 12a). This is due to the growing influence of the quadrature component. With decreasing dipole separation, the onset of this effect occurs at higher resistivities. For the range of parameters considered, CR and DC potentials are identical in magnitude only above resistivities of $\rho=10 \Omega \mathrm{m}$. For smaller resistivities, the influence of the quadrature component is significant and a resistivity estimate based upon the magnitude would be inadequate. The phase curves reflect this transition (Figure 12b). For high resistivities and large separations, the phase angle is close to zero, i.e. the observed potential is in phase with the injected current. Towards low resistivities however, phase shifts occur and the response eventually moves into quadrature.

Relative permittivity.-The sensitivity of the CR technique towards relative permittivity (dielectric constant) is only marginal at audio frequencies. For the selected frequency of $15 \mathrm{kHz}$ and the range of parameter values considered in this study, we have found the variation of the complex potential with relative permittivity to be negligible in the range between $\varepsilon_{r}=1$ (free space) and $\varepsilon_{r}=80$ (water). Previous 
studies have shown that a measurable variation of the CR transfer impedance with $\varepsilon_{r}$ at audio frequencies can be obtained in highly resistive environments only (Grard and Tabbagh, 1991; Tabbagh et al., 1993).

\section{EXPERIMENTAL VALIDATION}

In the following we describe a set of basic field-scale experiments undertaken with a newly developed CR instrument to validate the predictions of the electrostatic formalism. The so-called CRI (aaacitive Resistivity Imaging) system has been designed and built by the British Geological Survey and is a straightforward implementation of the theoretical concept of an electrostatic quadrupole. Its major improvement over previous implementations of CR technology is that it is capable of measuring the full complex transfer impedance across pairs of capacitive sensors without the need for a fixed reference potential. Its electronic design is based upon modern signal detection technology. The CRI system is designed for synchronous acquisition from multiple receiver channels and can perform both static and dynamic CR measurements (Kuras, 2002).

Experimental approach.-The following experimental approach was pursued: in an approximately homogeneous geological setting with known electrical properties, the complex transfer impedance was measured in a number of elementary surveys and compared with the predicted response for a homogeneous halfspace model calculated with the electrostatic formalism. A test area on the site of BGS headquarters in Keyworth (Nottinghamshire, UK) was selected for this purpose. On this site, nearsurface Mercia Mudstone deposits constitute an approximately uniform and isotropic electrical background with average resistivities of between $20 \Omega \mathrm{m}$ and $25 \Omega \mathrm{m}$ within the top few meters of ground. Two classes of experiments were carried out:

1. Moveout surveys were conducted to analyze the variation of the transfer impedance with distance and to assess the practicability of CR measurements 
across a range of signal-to-noise conditions.

2. Directional surveys were conducted to analyze the variation of the transfer impedance with azimuth between transmitter and receiver and to verify the dipolar character of the potential distribution.

All measurements were performed with the BGS prototype CRI system using platewire dipoles. These comprised stainless steel plates with dimensions $0.25 \mathrm{~m} \times 0.25 \mathrm{~m}$ and $1 \mathrm{~mm}$ thickness, laminated in plastic film for insulation. The surveys were conducted with a static setup, i.e. sensors were placed on the ground surface and current and potential readings taken at fixed positions. The results were then used to calculate $|Z|$ and $\operatorname{Arg}(Z)$. Measured data were compared with the predicted response for a homogeneous halfspace model calculated with the electrostatic formalism. The two unknown parameters in the experiments were resistivity and sensor elevation. To assess the sensitivity of the results with respect to either parameter, the variation of the potential with both resistivity and sensor elevation was considered. It was anticipated that average resistivities of $20 \Omega \mathrm{m} \leq \rho \leq 25 \Omega \mathrm{m}$ would be observed, and that the effective sensor elevation $h$ would be of the order of a few millimeters.

\section{Moveout surveys}

For this type of experiment the transmitter dipole remained at a fixed position while the receiver dipole was progressively moved away. In a laterally homogeneous environment, this procedure is equivalent to a centered resistivity sounding, but reduces the necessary array movements. Unit dipoles and injection currents of the order of $10 \mathrm{~mA}$ were employed throughout the experiment. Static measurements of the injected current and the in-phase and quadrature components of the observed potential were made, ranging from the smallest separation possible up to the maximum separation for which a stable measurement could be achieved. One hundred 
automated readings were taken at each location at a rate of $10 \mathrm{~Hz}$ and then stacked to obtain mean and standard deviation. The transfer impedance was calculated for each separation in order to make the results independent of possible current fluctuations during the experiment.

Here we discuss the results of a survey in which current and potential dipoles were arranged in equatorial configuration. In this geometry, the minimum dipole separation is limited only by the physical dimensions of the plates, hence a minimum separation of $0.3 \mathrm{~m}$ between the dipole centers could be employed. Separations were increased stepwise so that distances were distributed approximately evenly on a logarithmic scale. A maximum dipole separation of $12 \mathrm{~m}$ was found to be practical under the given circumstances, although it is likely that larger separations could have been achieved with a greater transmitter dipole moment, i.e. the use of larger injection currents or longer dipoles. A more resistive environment would also allow larger separations to be used.

The results for the equatorial survey are summarized in Figures 13 and 14. Magnitude and phase of the measured transfer impedance (colored symbols) are shown as a function of dipole separation together with the calculated curves for a homogeneous halfspace model. Statistical errors were found to be within the radius of the circles. In Figure 13, resistivity is the free model parameter while array elevation $h$ is kept constant at $0.5 \mathrm{~mm}$. The model resistivities cover a range of values from a conductive $(1 \Omega \mathrm{m})$ to a resistive regime $(1000 \Omega \mathrm{m})$, including the expected value of $20 \Omega \mathrm{m}$. Figure 14 shows the same measured data, but the free model parameter is now elevation, while resistivity is assumed constant at $20 \Omega \mathrm{m}$. Here, model elevations range from $0.1 \mathrm{~mm}$ to a maximum of $10 \mathrm{~mm}$, including the expected value of $0.5 \mathrm{~mm}$. Greater elevations are not realistic as the injected current would be limited by high impedances in the transmitter output circuit.

The clear vertical separation of the model curves in Figure 13a reflects the sensitivity of the magnitudes to resistivity. Magnitudes are much less affected by variations 
in elevation (Figure 14a) and a significant effect can be observed only for $h \geq 5 \mathrm{~mm}$. Under practical conditions, the magnitude measurement is therefore likely to be much less sensitive to array elevation than to resistivity. In direct contrast, the phase is highly sensitive to elevation (Figure 14b), but to a lesser extent to resistivity (Figure 13b), at least for separations greater than the dipole length $(r>l)$. The sensitivity of the phase to elevation is a consequence of the sensitivity of the quadrature component of $Z$ to the geometric factor $K^{\mathrm{ES}}$.

Optimal agreement between the measured data and the theoretical curves is observed for a resistivity of $\rho=20 \Omega \mathrm{m}$ (black solid curve in Figure 13) and an elevation of $h=0.5 \mathrm{~mm}$ (red solid curve in Figure 14). For the magnitudes, agreement with respect to both resistivity (Figure 13a) and elevation (Figure 14a) is best for separations between $0.4 \mathrm{~m}$ and $8 \mathrm{~m}$. In that interval, measured magnitudes follow the optimal curve very closely. For $r<0.4 \mathrm{~m}$ the measured magnitude exceeds the theoretical value by a significant margin. This effect is most likely to be due to the breakdown of the point-pole concept for finite-size plates at such small separations. For a plate width of $0.25 \mathrm{~m}$ and a dipole separation of $0.3 \mathrm{~m}$, the actual separation between the edges of the transmitter and receiver plates is only $5 \mathrm{~cm}$. This discrepancy leads to an overestimation of the measured magnitude compared to the theoretical value. At separations greater than $8 \mathrm{~m}$, the measured magnitude deviates from the optimum model curve towards significantly higher values. Considering the smooth nature of the deviation, it is more likely to be due to a genuine physical effect rather than a systematic error introduced by the experimental procedure. It can be explained by considering the associated phase response.

For the phases, measured data fit the model curves best between $0.4 \mathrm{~m}$ and $3 \mathrm{~m}$. A pronounced increase in phase is observed towards smaller separations $(r<0.7 \mathrm{~m})$. This effect is predicted by the quasi-static model, but to our knowledge has never been confirmed experimentally. For $r<0.4 \mathrm{~m}$ the measured phase exceeds the theoretical value in the same fashion as the magnitude. Phase values decrease with 
increasing separations and remain negligible for $0.7 \mathrm{~m}<r<3 \mathrm{~m}$. However, instead of maintaining the in-phase behavior as predicted by the quasi-static model, phase values begin to increase. The same increase was also observed in the magnitudes, where the effect is pronounced only at larger separations $(r>8 \mathrm{~m})$. Phases increase smoothly for $r>3 \mathrm{~m}$ and reach values of $|\varphi|>\pi / 4$ beyond $10 \mathrm{~m}$. We attribute this phenomenon to inductive effects becoming increasingly relevant in the relatively conductive environment, as previously suggested by Benderitter et al. (1994) and Tabbagh and Panissod (2000). The induction number (equation 24) is approximately 0.105 at $r=2 \mathrm{~m}$ and 0.529 at $r=10 \mathrm{~m}\left(f=14.2 \mathrm{kHz}, \sigma=0.05 \mathrm{Sm}^{-1}\right)$, indicating that Benderitter's criterion (25) is marginal beyond $r=2 \mathrm{~m}$. Under the given circumstances in a relatively conductive environment, the quasi-static approximation has reached its limit and for longer offsets the response is more adequately described by electromagnetic theory.

\section{Directional surveys}

In the second set of experiments the directional variation of the CR transfer impedance was examined. For these surveys, the location of the current dipole remained fixed while the potential dipole was rotated around the current dipole at a constant separation, i.e. on a circular arc, maintaining a constant mutual orientation between the two dipoles. Two complementary configurations are possible, in which current and potential dipoles have either parallel or perpendicular orientation. This type of survey was expected to give additional information about the shape of the source field generated by the current dipole, which is anticipated to have typical dipolar characteristics.

In similar fashion to the moveout surveys, a series of static measurements were carried out at a range of azimuth angles. Unit dipoles at a separation of $5 \mathrm{~m}$ between midpoints were employed. Theoretical responses for the homogeneous halfspace model 
were calculated as before. For a directional survey, the complex transfer impedance can be regarded as a periodic function of the azimuth $\phi$. Due to symmetry it is therefore sufficient to restrict the survey to a quarter-circle, i.e. $\phi \in\left[0^{\circ} \ldots 90^{\circ}\right]$. The surveys were carried out at a different time of the year than the moveout surveys, hence a slightly elevated background resistivity $(\rho \approx 25 \Omega \mathrm{m})$ was anticipated.

Here we discuss the results of a survey with dipoles in a parallel configuration (Figure 15). Starting from an equatorial position $\left(\phi=0^{\circ}\right)$, the azimuth $\phi$ was increased in steps of $5^{\circ}$ until an axial or inline position $\left(\phi=90^{\circ}\right)$ was reached. The results of this survey are summarized in Figures 16 and 17, which are plotted as a function of azimuth on a linear scale. In Figure 16, resistivity is the free model parameter while elevation is kept constant at $1 \mathrm{~mm}$. The same measured data are shown in Figure 17, but now elevation is the free model parameter while resistivity is kept constant at $25 \Omega \mathrm{m}$. An angular interval of $180^{\circ}$, centered around an azimuth of $\phi=45^{\circ}$, is displayed to capture the periodicity of the response. The magnitude has two distinct maxima, an absolute maximum at $\phi=90^{\circ}$ and a secondary maximum at $\phi=0^{\circ}$. These are consistent with the interpretation of the axial configuration as the position of maximum coupling, while the equatorial configuration is associated with a local maximum only. Between the two maxima the magnitude has a zero and the phase changes quadrants $\left(\phi \approx 36^{\circ}\right)$. In similar fashion to the moveout results, the variation of the magnitude with resistivity (Figure 16a) is strong while the effect of elevation on magnitude (Figure 17a) is much weaker. In contrast, the variation of the phase with resistivity (Figure 16b) is relatively weak, while its variation with elevation (Figure 17b) is stronger.

Due to the change in seasonal conditions, the optimal fit between measured data and calculated model curves is now observed at $\rho=25 \Omega \mathrm{m}$. The agreement between measured magnitudes and the optimal curve at $\rho=25 \Omega \mathrm{m}$ (Figure 16a) is remarkably good. The minimum near $\phi=36^{\circ}$ is reproduced by the experimental data, however at a slightly lower azimuth. Figure 17a shows that measured magnitudes match a 
range of possible elevations smaller than $5 \mathrm{~mm}$. Interpretation of the phases must be undertaken with caution. At a dipole separation of $5 \mathrm{~m}$, modeled phases are likely to be very small, however the moveout survey has demonstrated that measured phases at that distance may already deviate significantly from the quasi-static model. The phase model curves were therefore not expected to fit the data particularly well. However, phase values were generally found to be of the expected order and follow the change in quadrants predicted by the model curves. (Figures 16b, 17b).

\section{ESTIMATION OF APPARENT RESISTIVITY}

The electrostatic formalism predicts the CR response in the form of the transfer impedance, based upon an electrical model of the subsurface and geometric parameters of the measurement. A practical field technique must solve the reverse problem, i.e. apparent resistivity must be derived from a measurement of the transfer impedance. In DC resistivity, the determination of apparent resistivity is trivial as all calculations involve real values and resistivity of the homogeneous halfspace is the only model parameter. In CR however, the measured transfer impedance is generally complex and the electrostatic formalism has more than one model parameter, namely resistivity, relative permittivity and array elevation. We will demonstrate in the following that a unique solution exists also for the CR problem and that resistivity can be estimated from magnitude and phase (or in-phase and quadrature components) of the observed transfer impedance.

\section{The reverse problem}

The factor $\alpha$ (equation 20) is found to be a convenient starting point for the following calculation. The complex expression can be split into real and imaginary parts: 


$$
\begin{aligned}
\operatorname{Re} \alpha & =\frac{\rho^{2} \omega^{2} \varepsilon_{0}^{2}\left(\varepsilon_{r}^{2}-1\right)+1}{\rho^{2} \omega^{2} \varepsilon_{0}^{2}\left(\varepsilon_{r}+1\right)^{2}+1} \\
\operatorname{Im} \alpha & =\frac{-2 \rho \omega \varepsilon_{0}}{\rho^{2} \omega^{2} \varepsilon_{0}^{2}\left(\varepsilon_{r}+1\right)^{2}+1} .
\end{aligned}
$$

Solving equations (41a) and (41b) for $\rho$ and $\varepsilon_{r}$, we obtain

$$
\begin{aligned}
\rho^{2} & =\frac{1-\operatorname{Re} \alpha}{\omega^{2} \varepsilon_{0}^{2}\left(\left(\varepsilon_{r}+1\right)^{2} \operatorname{Re} \alpha-\varepsilon_{\mathrm{r}}^{2}+1\right)} \\
\varepsilon_{r} & =\sqrt{-\frac{2 \rho \omega \varepsilon_{0}+\operatorname{Im} \alpha}{\rho^{2} \omega^{2} \varepsilon_{0}^{2} \operatorname{Im} \alpha}}-1 .
\end{aligned}
$$

Mutual insertion eliminates the interdependence and a relation for resistivity is obtained that is a function of $\alpha$ only:

$$
\rho=-\frac{1}{2 \omega \varepsilon_{0}}\left(\frac{(1-\operatorname{Re} \alpha)^{2}}{\operatorname{Im} \alpha}+\operatorname{Im} \alpha\right)
$$

In similar fashion, an expression for relative permittivity can be found:

$$
\varepsilon_{r}=\frac{2|1-\operatorname{Re} \alpha|}{(1-\operatorname{Re} \alpha)^{2}+(\operatorname{Im} \alpha)^{2}}-1
$$

This is an important result because it shows that the quasi-static formulation of the complex transfer impedance formally contains independent information about both $\rho$ and $\varepsilon_{r}$. However, as previously discussed, it is unlikely that reliable estimates of $\varepsilon_{r}$ can be obtained by low-frequency electromagnetic methods. Determination of $\varepsilon_{r}$ with the CR technique may be possible at higher frequencies and in highly resistive geological environments (Tabbagh et al., 1993).

Determination of $\alpha$ from a measured impedance.-The reverse problem is now reduced to the determination of $\alpha$ from a practical impedance measurement. The transfer impedance $Z$ can be expressed by the current $I(t)=\hat{I} e^{i \omega t}$ injected across the current dipole $\left(C_{1}, C_{2}\right)$ and the voltage $U(t)=\hat{U} e^{i \omega t} e^{i \varphi}$ observed at the potential dipole $\left(P_{1}, P_{2}\right)$, where $\varphi$ is the phase shift between the two signals:

$$
Z=\frac{U}{I}=\frac{\hat{U} e^{i \varphi}}{\hat{I}}=\frac{\hat{U}(\cos \varphi+i \sin \varphi)}{\hat{I}}=\hat{Z}(\cos \varphi+i \sin \varphi) .
$$


In a practical measurement, $\hat{U}, \hat{I}$ and $\varphi$ must be determined. $\hat{U}$ and $\hat{I}$ can be either true amplitudes or RMS values, as long as both are of the same type. Reordering equation (23) for $\alpha$ yields

$$
\alpha=\frac{1}{K^{\mathrm{ES}}}\left(1-\frac{Z}{Z_{0}}\right),
$$

and after insertion

$$
\alpha=\frac{1}{K^{\mathrm{ES}}}\left(1-i \omega C_{0} \hat{Z}(\cos \varphi+i \sin \varphi)\right)
$$

An expression for apparent resistivity.-After separating $\alpha$ into in-phase and quadrature components, the measured apparent resistivity (43) finally becomes

$$
\begin{gathered}
\rho_{a}=-\frac{1}{2 \omega \varepsilon_{0}}\left(\frac{(1-\operatorname{Re} \alpha)^{2}}{\operatorname{Im} \alpha}+\operatorname{Im} \alpha\right) \\
\operatorname{Re} \alpha=\frac{1}{\mathrm{~K}^{\mathrm{ES}}}\left(1+\omega \mathrm{C}_{0} \hat{\mathrm{Z}} \sin \varphi\right)=\frac{1}{\mathrm{~K}^{\mathrm{ES}}}\left(1+\omega \mathrm{C}_{0} \operatorname{Im} \mathrm{Z}\right) \\
\operatorname{Im} \alpha=-\frac{1}{\mathrm{~K}^{\mathrm{ES}}} \cdot \omega \mathrm{C}_{0} \hat{\mathrm{Z}} \cos \varphi=-\frac{1}{\mathrm{~K}^{\mathrm{ES}}} \cdot \omega \mathrm{C}_{0} \operatorname{Re} \mathrm{Z},
\end{gathered}
$$

and a unique solution is found for the reverse problem. These three equations represent an expression for apparent resistivity as derived from a capacitive measurement under quasi-static conditions. It is evident that $\rho_{a}=\rho_{a}(\hat{Z}, \varphi)$, i.e. $\rho_{a}$ is now a function of magnitude and phase of the measured transfer impedance.

\section{RELATIONSHIP BETWEEN CR AND CONVENTIONAL METHODS}

DC resistivity.-The different expressions for estimating resistivity with CR (equation 48a) and conventional DC (equation 1) characterize the relationship between the two techniques. In the case of $\mathrm{CR}$, it is found that a number of conditions apply that further simplify equation (48a). Closer inspection of the factor $\alpha$ (equation 20) shows that Re $\alpha \approx 1$ at low induction numbers (Grard, 1990; Kuras, 2002). 
Under such circumstances, and if the quadrupole is close to the surface $\left(K^{\mathrm{ES}} \approx 1\right)$, the quadrature component of $Z$ vanishes (equation $48 \mathrm{~b}$ ). The CR response can then be expected to be in phase. As a consequence, equation (48a) reduces to

$$
\rho_{a} \approx-\frac{\operatorname{Im} \alpha}{2 \omega \varepsilon_{0}} \approx \frac{C_{0}}{2 \varepsilon_{0}} \operatorname{Re} \mathrm{Z}
$$

and together with the definition of $C_{0}(14)$ one obtains

$$
\rho_{a} \approx \frac{\operatorname{Re} \mathrm{U}}{\hat{I}} \cdot K^{\mathrm{DC}}
$$

i.e. the phase-sensitive apparent resistivity (48a) reduces to the classical DC expression for the in-phase component of the transfer impedance. The electrostatic formalism can therefore be regarded as a generalization of conventional DC theory (Tabbagh et al., 1993).

We should mention that a number of existing CR instruments operate on the basis of the assumption that the observed potential is entirely in phase with the injected current, so that it is sufficient to measure its magnitude only. However, ignoring the phase sensitivity of the potential will only be justified if (a) the quasi-static condition is maintained over all survey measurement points and (b) the geometric arrangement of the sensors is such that the potential dipole is neither too close to nor too distant from the current dipole. Condition (a) may become increasingly marginal as increasingly conductive conditions are encountered. It is also important to recognize that the phase dependence of a $\mathrm{CR}$ measurement is primarily due to the capacitive coupling mechanism and the geometry of the sensor array; it is not directly associated with a subsurface material property. In this respect, the phase dependence of CR measurements is totally distinct from phase rotations obtained by induced polarization (IP) or complex resistivity measurements, which arise due to electrical polarization in the subsurface.

Electromagnetic ground conductivity.-An adaptation of the classical electromagnetic coil-coil method of Slingram operates at low induction numbers and is often 
referred to as "ground conductivity". The method is based on the mutual inductive coupling of two coils and is used extensively in environmental and engineering applications.

Under quasi-static conditions, i.e. at low induction numbers, it can be shown (McNeill, 1980) that the mutual coupling ratio $Z / Z_{0}$ as the fundamental system response is directly proportional to conductivity. The apparent resistivity is then given as

$$
\frac{1}{\rho_{a}}=\sigma_{a} \approx \frac{4}{\omega \mu_{0} s^{2}} \operatorname{Im}\left(\frac{\mathrm{Z}}{\mathrm{Z}_{0}}\right)
$$

i.e. practical instruments can be calibrated to display a conductivity reading directly. Comparison with equation (50) shows that the $\mathrm{CR}$ and ground conductivity techniques can be regarded as complementary; an inductively coupled measurement provides information about subsurface resistivity in the quadrature of the response function, while the equivalent information for a capacitively coupled measurement is contained in the in-phase component.

\section{CONCLUSIONS}

In this study we have set out the basis for making resistivity measurements of the near surface based on a capacitive coupling mechanism. We have demonstrated that different realizations of the capacitive resistivity technique are conceivable and that the theoretical formulation of CR is invariably linked with the practical design of capacitive sensors. However, the classical DC resistivity measurement can be emulated by the CR technique and well-known DC interpretation schemes are applicable to CR data, provided that sensor design and geometry are carefully considered. The fundamental concept of point poles (i.e. infinitesimally small electrodes) is transferred to CR theory by means of the electrostatic formalism, which allows the transfer impedance between current and potential dipoles to be complex. 
For the purposes of practical measurements, we have demonstrated how capacitive plate-wire combinations can be employed to simulate point poles. The surface area of the sensor determines its capacitance, which was shown to be the relevant quantity that ensures adequate coupling between sensor and the ground. A quadrupole of capacitive plate-wire combinations was found to measure complex potentials consistent with those expected for a four-point array.

Using quasi-static theory, we have carried out a detailed analysis of the properties of the complex transfer impedance and conclude that a realistic range of parameters exists that allows successful operation of practical CR instruments under quasi-static conditions. Experimental validation of the predicted $\mathrm{CR}$ response was carried out using a recently developed prototype CR instrument. Based on the results obtained, we propose that three different ranges of separation between current and potential dipoles should be distinguished, namely

1. a normal or intermediate zone in which measured data can be accurately modeled by quasi-static theory,

2. a near zone in which plate size becomes large compared with array dimensions so that the transfer impedance is overestimated, and

3. a far zone in which the quasi-static approximation breaks down and full electromagnetic theory is required to describe the response of the CR array.

We have provided a quasi-static formulation of apparent resistivity specific to the $\mathrm{CR}$ technique and demonstrated that the phase-sensitive expression reduces to the classical DC formula for the in-phase component of the transfer impedance. The electrostatic formalism therefore represents a generalization of conventional DC theory for a capacitive coupling mechanism. A comparison of CR with the inductively coupled ground conductivity technique suggests that the two techniques may be regarded as complementary. 


\section{ACKNOWLEDGMENTS}

The authors gratefully acknowledge the thorough reviews provided by Jeff Johnston, Michel Chouteau, Klaus Spitzer, Vladimir Timofeev and associate editor Louise Pellerin. This paper is published with the permission of the Executive Director of the British Geological Survey (NERC).

\section{REFERENCES}

Benderitter, Y., Jolivet, A., Mounir, A., and Tabbagh, A., 1994, Application of the electrostatic quadripole to sounding in the hectometric depth range: Journal of Applied Geophysics, 31, 1-6.

Bristow, Q., and Mwenifumbo, C. J., 1998, Evaluation of a russian-designed borehole resistivity probe based on a capacitive principle: Eastern Canada and national and general programs, Current Research Geological Survey of Canada, 1998-D, 65-73.

Chouteau, M., Vallieres, S., and Miralles, M., 2004, Assessment of continuous resistivity profiling for the characterization of paved roads: Eos Transactions American Geophysical Union, 85, no. 17, Joint Assembly Supplement, Abstract NS43A-03.

Cook, J. C., 1956, An electrical crevasse detector: Geophysics, 21, 1055-1070.

Cottineau, L. M., Desmas, M., Lagabrielle, R., Leroux, V., Palma Lopes, S., and Sorin, J. L., 2000, Capacitive contact geoelectrical equipment development for borehole environmental and engineering applications: 6th Meeting of the Environmental and Engineering Geophysical Society - European Section, Expanded Abstracts.

Grard, R., and Tabbagh, A., 1991, A mobile four-electrode array and its application to the electrical survey of planetary grounds at shallow depths: Journal of Geophysical Research, 96, no. B3, 4117-4123. 
Grard, R., 1990, A quadrupolar array for measuring the complex permittivity of the ground: application to Earth prospection and planetary exploration: Measurement Science \& Technology, 1, 295-301.

Kuras, O., 2002, The capacitive resistivity technique for electrical imaging of the shallow subsurface: Ph.D. thesis, University of Nottingham.

Lee, S. K., Cho, S.-J., Song, Y., and Chung, S.-H., 2002, Capacitively-coupled resistivity method - applicability and limitation: MULLI-TAMSA (Geophysical Exploration), $\mathbf{5}$, no. $1,23-32$.

McNeill, J. D., 1980, Electromagnetic terrain conductivity measurement at low induction numbers: Technical note TN-6, Geonics Ltd.

Møller, I., 2001, OhmMapper field tests at sandy and clay till sites in Denmark: 7th meeting of the Environmental and Engineering Geophysical Society - European Section, Birmingham, Proceedings, 100-101.

Mwenifumbo, C. J., and Bristow, Q., 1999, Field evaluation of a new borehole resistivity probe using capacitive electrodes: Symposium on the Application of Geophysics to Engineering and Environmental Problems (SAGEEP), Proceedings, 859.

Panissod, C., Dabas, M., Hesse, A., Jolivet, A., Tabbagh, J., and Tabbagh, A., 1998, Recent developments in shallow-depth electrical and electrostatic prospecting using mobile arrays: Geophysics, 63, no. 5, 1542-1550.

Parasnis, D. S., 1997, Principles of Applied Geophysics: Chapman \& Hall, 5th edition.

Pellerin, L., Groom, D., and Johnston, J., 2003, Multi-receiver OhmMapper survey over a former fuel tank site: 9th Meeting of the Environmental and Engineering Geophysical Society - European Section, Proceedings, O-078. 
Ringhandt, A., and Wagemann, H. G., 1993, An exact calculation of the 2-dimensional capacitance of a wire and a new approximation formula: IEEE Transactions on Electron Devices, 40, 1028-1032.

Sakurai, T., and Tamaru, K., 1983, Simple formulas for two- and three-dimensional capacitances: IEEE Transactions on Electron Devices, 30, 183-185.

Shima, H., Texier, B., Kobayashi, T., and Hasegawa, N., 1995, Fast imaging of shallow resistivity structures using a multichannel capacitive electrode system: 65th Annual International Meeting, SEG, Expanded Abstracts, 377-380.

Shima, H., Sakashita, S., and Kobayashi, T., 1996, Developments of non-contact data acquisition techniques in electrical and electromagnetic explorations: Journal of Applied Geophysics, 35, 167-173.

Smythe, W. R., 1950, Static and Dynamic Electricity: McGraw-Hill, 2nd edition.

Tabbagh, A., and Panissod, C., 2000, 1D complete calculation for electrostatic soundings interpretation: Geophysical Prospecting, 48, 511-520.

Tabbagh, A., Hesse, A., and Grard, R., 1993, Determination of electrical properties of the ground at shallow depth with an electrostatic quadrupole: field trials on archaeological sites: Geophysical Prospecting, 41, 579-597.

Telford, W. M., Geldart, L. P., and Sheriff, R. E., 1990, Applied Geophysics: Cambridge University Press, 2nd edition.

Timofeev, V. M., 1973, Experience in the use of high frequency electrical geophysical methods in geotechnical and geocryological field studies: 3rd International Conference on Permafrost, NAUKA, Proceedings, 238-247.

Timofeev, V. M., 1974, The employment of capacitively-coupled sensors in engineering and geological studies (in Russian): Ph.D. thesis, University of Moscow. 
Timofeev, V. M., 1978, Some peculiarities of surface capacitive antennas used in electrical geophysical techniques: Reference Information Issue 8/73, Gosstroi USSR, PNIIS.

Topp, G. C., Davis, J. L., and Annan, A. P., 1980, Electromagnetic determination of soil water content: Measurements in coaxial transmission lines: Water Resources Research, 16, no. 3, 574-582.

Wait, J. R., 1995, Comments on: Benderitter et al., Application of the electrostatic quadripole to sounding in the hectometric depth range: Journal of Applied Geophysics, 34, 79-80.

Yuan, C. P., and Trick, T. N., 1982, A simple formula for the estimation of the capacitance of two-dimensional interconnects in VLSI circuits: IEEE Electron Device Letters, 3, 391-393. 
TABLES

\begin{tabular}{|c|c|c|c|}
\hline \hline & Small & Typical & Large \\
\hline$\rho[\Omega \mathrm{m}]$ & 1 & 50 & 100000 \\
$\mathrm{f}[\mathrm{kHz}]$ & 1 & 15 & 100 \\
$\varepsilon_{r}$ & 1 & 3 & 80 \\
\hline$\Omega$ & $1.1 \cdot 10^{-7}$ & $1.7 \cdot 10^{-4}$ & 45.1 \\
\hline$B(L=1 \mathrm{~m})$ & 0.063 & 0.034 & 0.002 \\
\hline \hline
\end{tabular}

TABLE 1. Range of possible values for the generalized frequency $\Omega$ and associated induction numbers $B$ for a scale length of $L=1 \mathrm{~m}$. 


\section{FIGURES}

FIG. 1. Equivalent electrical circuit models of (a) a generic resistivity measurement, (b) DC resistivity and (c) capacitive resistivity. The generic model (Wait, 1995) shows the ground impedance $Z_{\text {Earth }}$ and the contact impedances $Z_{\mathrm{C} 1}, Z_{\mathrm{C} 2}$ (current injection) and $Z_{\mathrm{P} 1}, Z_{\mathrm{P} 2}$ (potential measurement). The contact impedances are predominantly ohmic in DC resistivity and predominantly capacitive in CR.

FIG. 2. Geometry of the four-electrode arrangement used for a DC resistivity measurement.

FIG. 3. Concept of the equivalent grounded dipole: inline dipole-dipole array composed of (a) capacitive line antennas and (b) equivalent grounded dipoles.

FIG. 4. Electrostatic point charge $Q$ near the interface between two media with dielectric permittivities $\varepsilon^{(I)}$ and $\varepsilon^{(I I)}$. The image charge $Q^{\prime}$ is located at the opposite side of the interface.

FIG. 5. Geometry of an electrostatic quadrupole in the general case. Point charges $+Q$ and $-Q$ are situated at $C_{1}$ and $C_{2}$ near the interface between two permittive media. Image charges are located at $C_{1}^{\prime}$ and $C_{2}^{\prime}$ on the opposite side of the interface and the difference in electrostatic potential is measured between $P_{1}$ and $P_{2}$.

FIG. 6. Conceptual model of a capacitive line antenna close to the ground surface.

FIG. 7. Conceptual model of a capacitive plate-wire combination close to the ground surface. 
FIG. 8. Variation of the normalized transfer impedance $Z /\left|Z_{0}\right|$ with generalized frequency $\Omega$ in magnitude (a) and phase (b) under quasi-static conditions. Results for five values of the geometric factor $K^{\mathrm{ES}}$ are shown.

FIG. 9. Variation of the complex potential with frequency in magnitude (a) and phase (b) under quasi-static conditions for a unit square capacitive array $(l=1 \mathrm{~m}$, $r=1 \mathrm{~m}$ ). Results for five values of resistivity are shown. Array elevation $h=1 \mathrm{~mm}$, relative permittivity $\varepsilon_{r}=10$, injected current $I=10 \mathrm{~mA}$. Magnitude values for an equivalent DC array are shown as dashed lines.

FIG. 10. Variation of the complex potential with dipole separation in magnitude (a) and phase (b) under quasi-static conditions for a capacitive array in equatorial configuration. Results for five values of resistivity are shown. Dipole length $l=1 \mathrm{~m}$, array elevation $h=1 \mathrm{~mm}, \varepsilon_{r}=10, f=15 \mathrm{kHz}, I=10 \mathrm{~mA}$. Magnitude values for an equivalent DC array are shown as dashed lines.

FIG. 11. Variation of the complex potential with array elevation in magnitude (a) and phase (b) under quasi-static conditions for a capacitive array in equatorial configuration. Results for five values of resistivity are shown. Dipole length $l=1 \mathrm{~m}$, dipole separation $r=1 \mathrm{~m}, \varepsilon_{r}=10, f=15 \mathrm{kHz}, I=10 \mathrm{~mA}$. Magnitude values for an equivalent DC array are shown as dashed lines.

FIG. 12. Variation of the complex potential with resistivity in magnitude (a) and phase (b) under quasi-static conditions for a capacitive array in equatorial configuration. Results for five values of the dipole separation $r$ are shown. Dipole length $l=1 \mathrm{~m}$, array elevation $h=1 \mathrm{~mm}, \varepsilon_{r}=10, f=15 \mathrm{kHz}, I=10 \mathrm{~mA}$. Magnitude values for an equivalent DC array are shown as dashed lines. 
FIG. 13. Complex transfer impedances for the moveout survey in equatorial configuration: comparison of measured data with quasi-static model in magnitude (a) and phase (b). Results for five values of resistivity are shown; model array elevation remained fixed at $h=0.5 \mathrm{~mm}$.

FIG. 14. Complex transfer impedances for the moveout survey in equatorial configuration: comparison of measured data with quasi-static model in magnitude (a) and phase (b). Results for five values of array elevation are shown; model resistivity remained fixed at $\rho=20 \Omega \mathrm{m}$.

FIG. 15. Directional survey in parallel configuration to determine the azimuthal variation of the transfer impedance.

FIG. 16. Transfer impedances for the directional survey in parallel configuration: comparison of measured data with quasi-static model in magnitude (a) and phase (b). Results for five values of resistivity are shown; model array elevation remained fixed at $h=1 \mathrm{~mm}$.

FIG. 17. Transfer impedances for the directional survey in parallel configuration: comparison of measured data with quasi-static model in magnitude (a) and phase (b). Results for five values of array elevation are shown; model resistivity remained fixed at $\rho=25 \Omega \mathrm{m}$. 
(a)
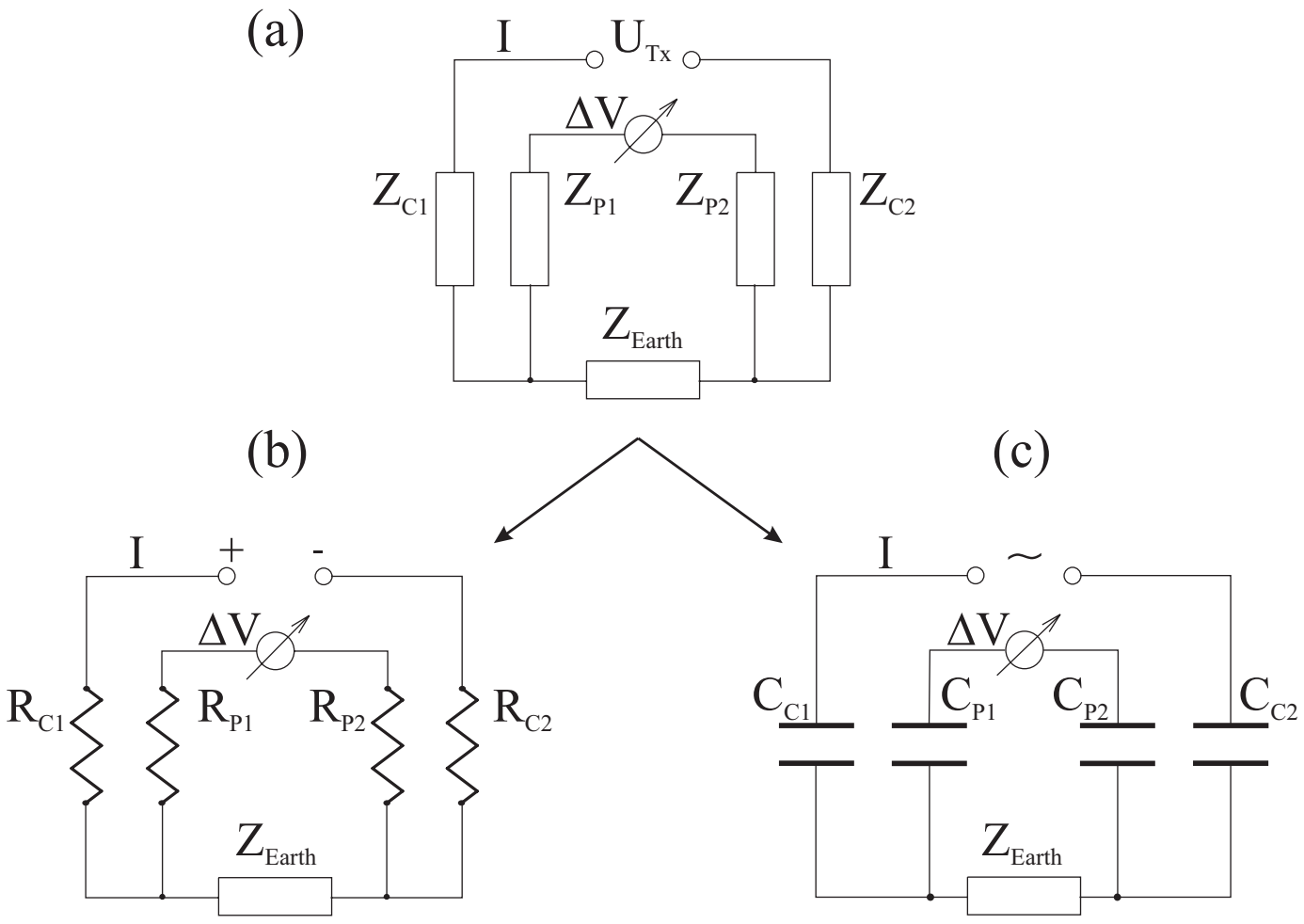

FIG. 1. Equivalent electrical circuit models of (a) a generic resistivity measurement, (b) DC resistivity and (c) capacitive resistivity. The generic model (Wait, 1995) shows the ground impedance $Z_{\text {Earth }}$ and the contact impedances $Z_{\mathrm{C} 1}, Z_{\mathrm{C} 2}$ (current injection) and $Z_{\mathrm{P} 1}, Z_{\mathrm{P} 2}$ (potential measurement). The contact impedances are predominantly ohmic in DC resistivity and predominantly capacitive in CR. 


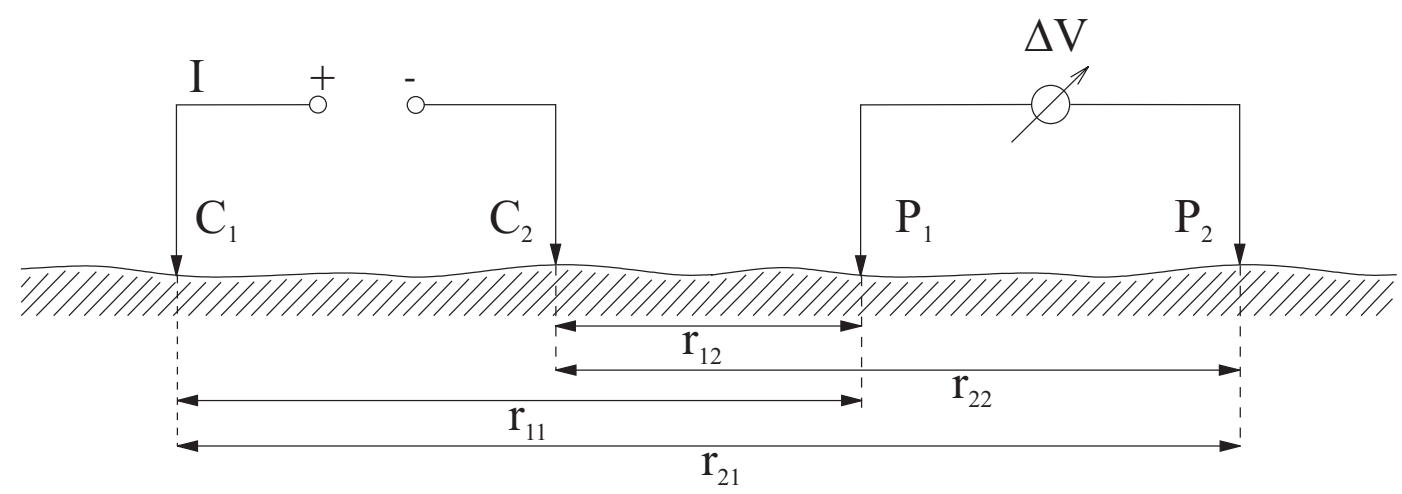

FIG. 2. Geometry of the four-electrode arrangement used for a DC resistivity measurement. 
Current dipole

(Tx)

(a)

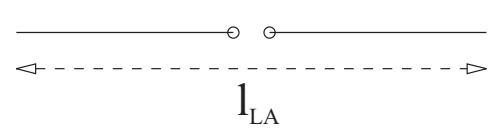

(b)

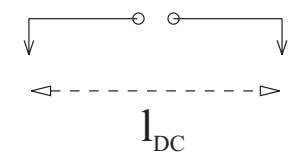

Potential dipole

$(\mathrm{Rx})$

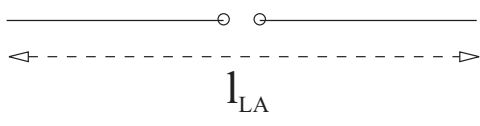

r

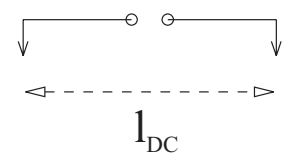

FIG. 3. Concept of the equivalent grounded dipole: inline dipole-dipole array composed of (a) capacitive line antennas and (b) equivalent grounded dipoles. 


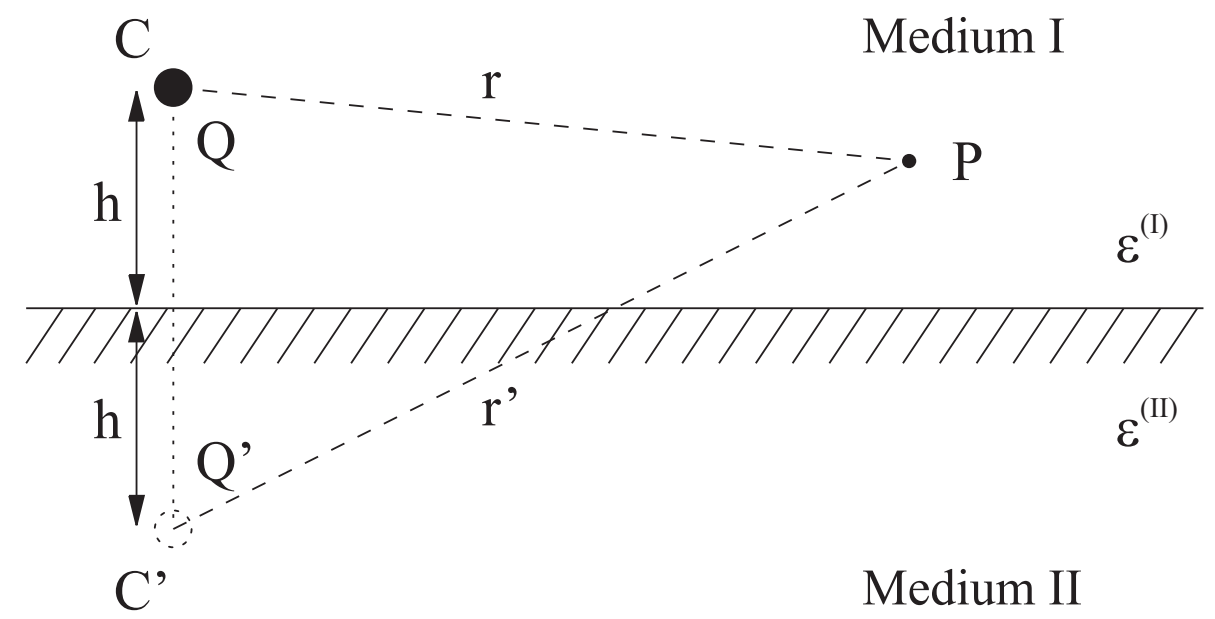

FIG. 4. Electrostatic point charge $Q$ near the interface between two media with dielectric permittivities $\varepsilon^{(I)}$ and $\varepsilon^{(I I)}$. The image charge $Q^{\prime}$ is located at the opposite side of the interface. 


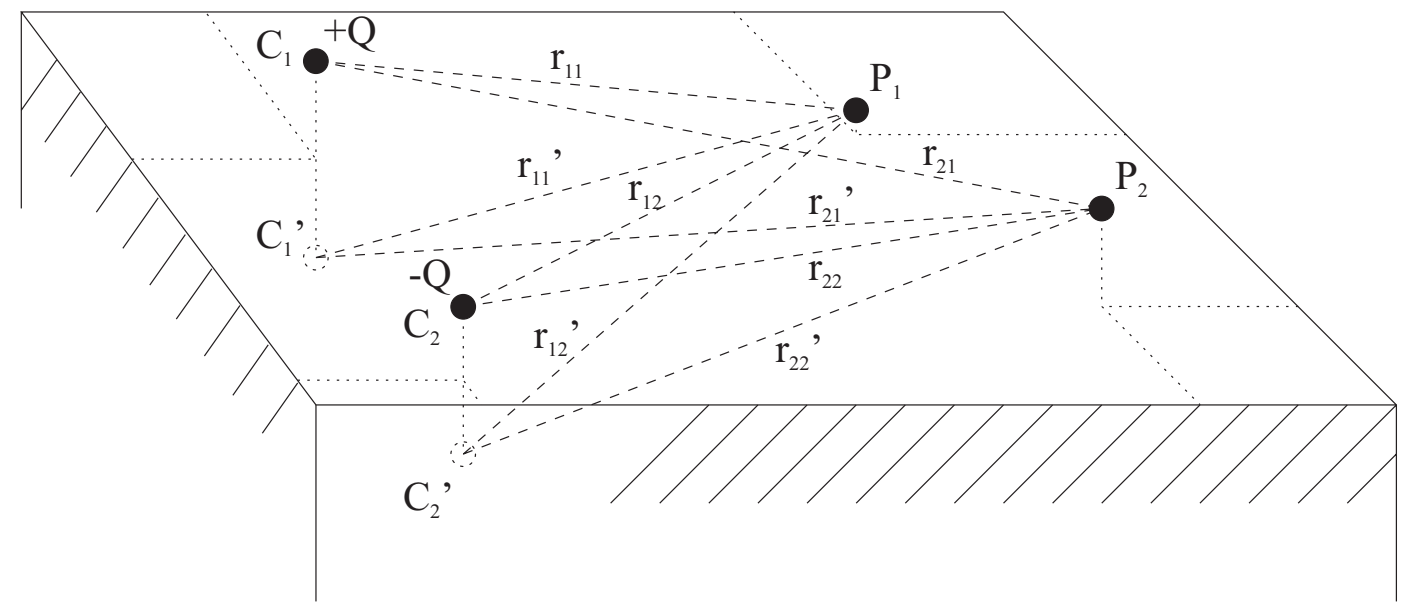

FIG. 5. Geometry of an electrostatic quadrupole in the general case. Point charges $+Q$ and $-Q$ are situated at $C_{1}$ and $C_{2}$ near the interface between two permittive media. Image charges are located at $C_{1}^{\prime}$ and $C_{2}^{\prime}$ on the opposite side of the interface and the difference in electrostatic potential is measured between $P_{1}$ and $P_{2}$. 


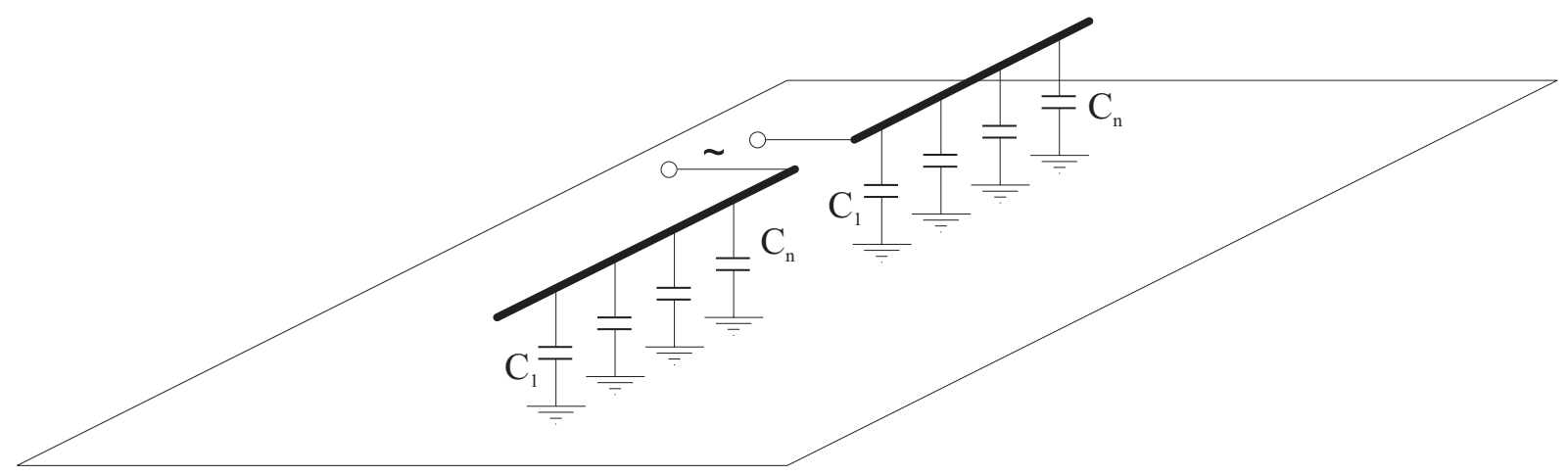

FIG. 6. Conceptual model of a capacitive line antenna close to the ground surface. 


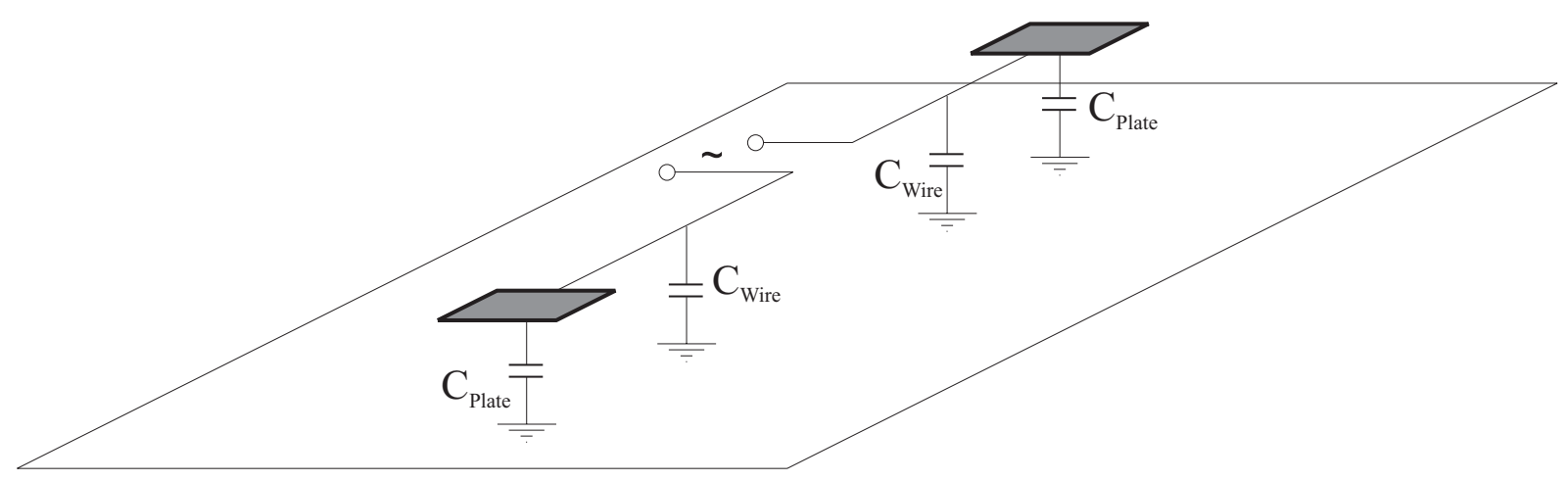

FIG. 7. Conceptual model of a capacitive plate-wire combination close to the ground surface. 
(a) Magnitude

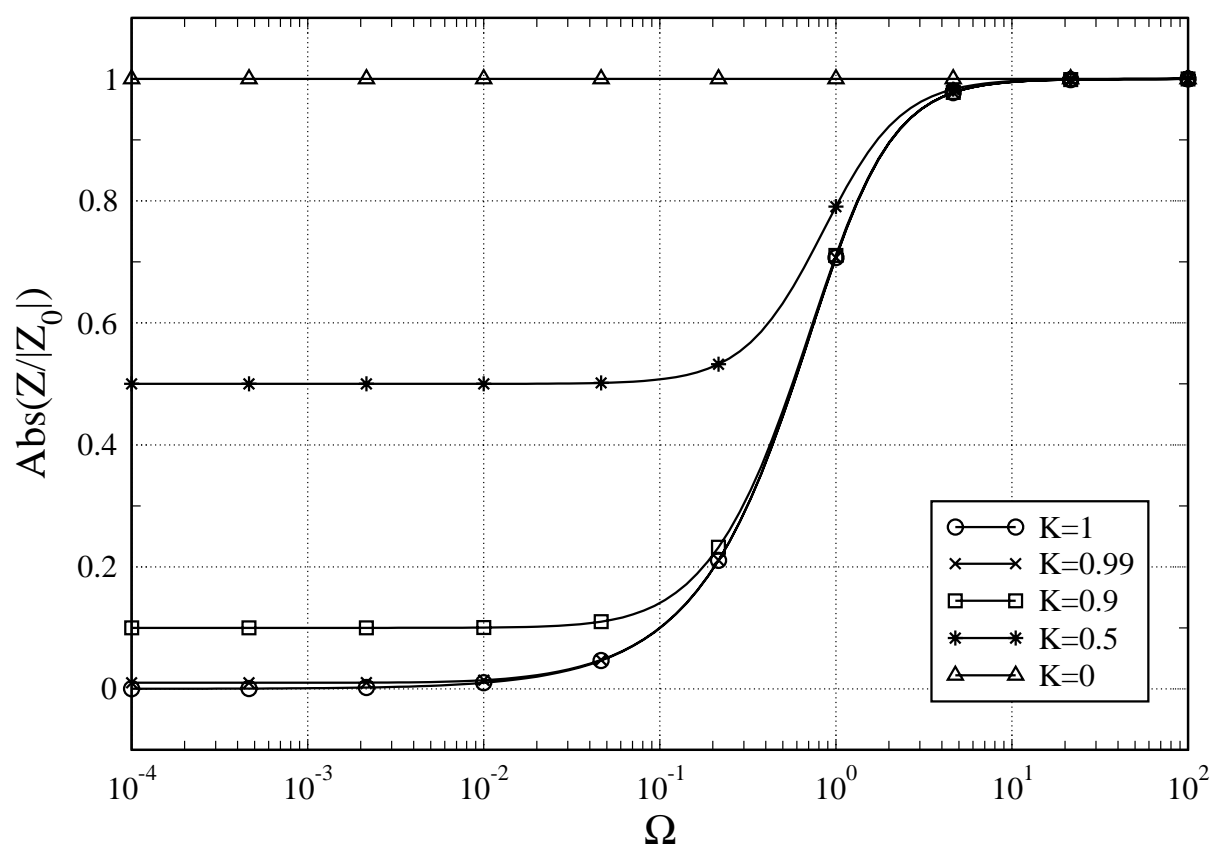

(b) Phase

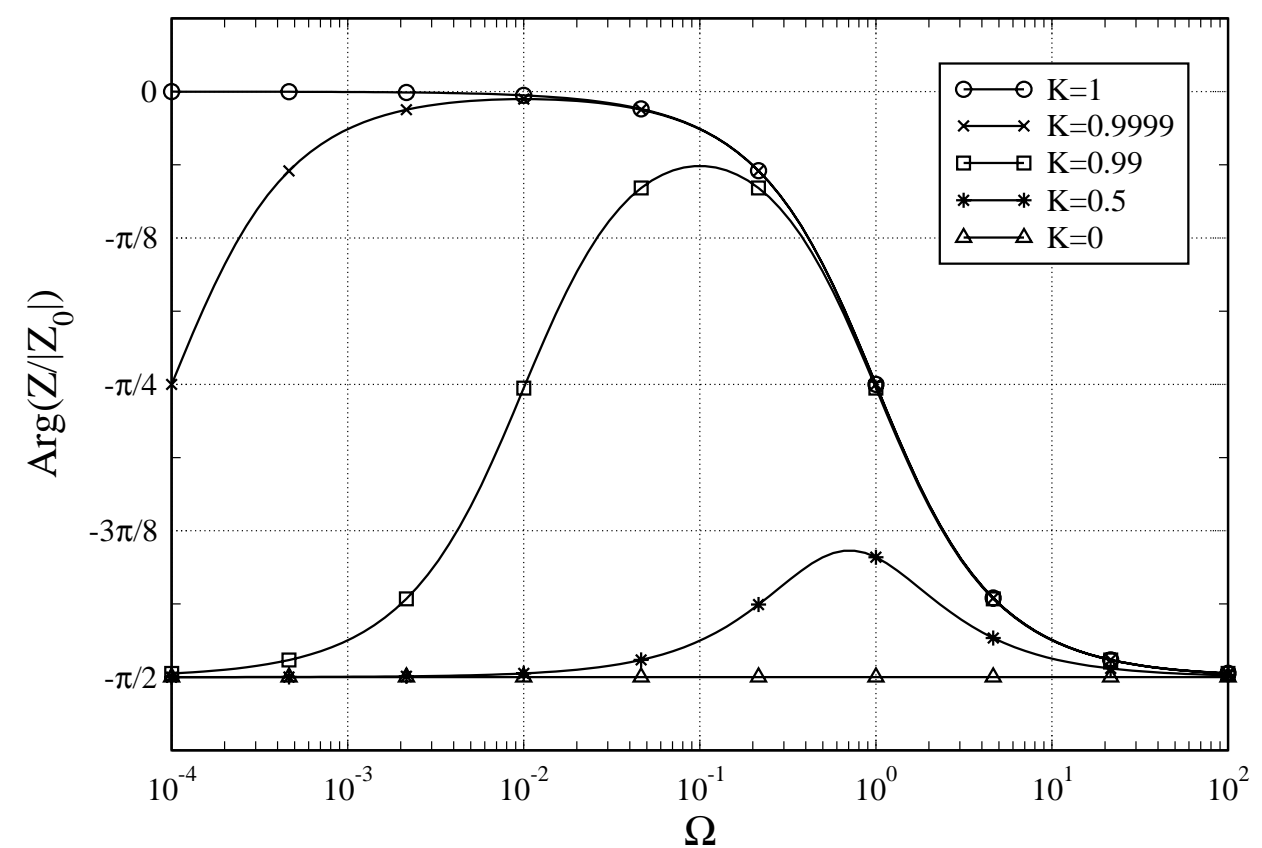

FIG. 8. Variation of the normalized transfer impedance $Z /\left|Z_{0}\right|$ with generalized frequency $\Omega$ in magnitude (a) and phase (b) under quasi-static conditions. Results for five values of the geometric factor $K^{\mathrm{ES}}$ are shown. 
(a) Magnitude

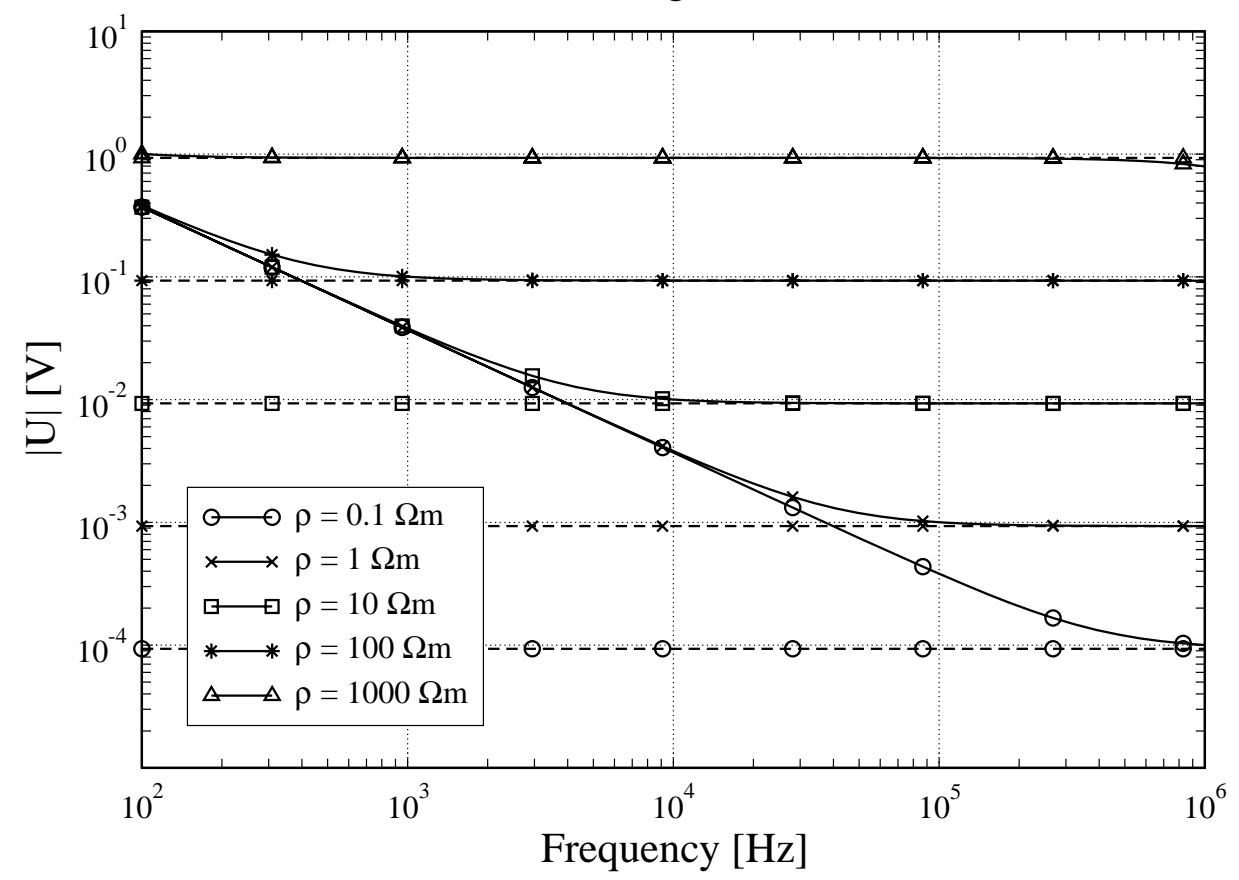

(b) Phase

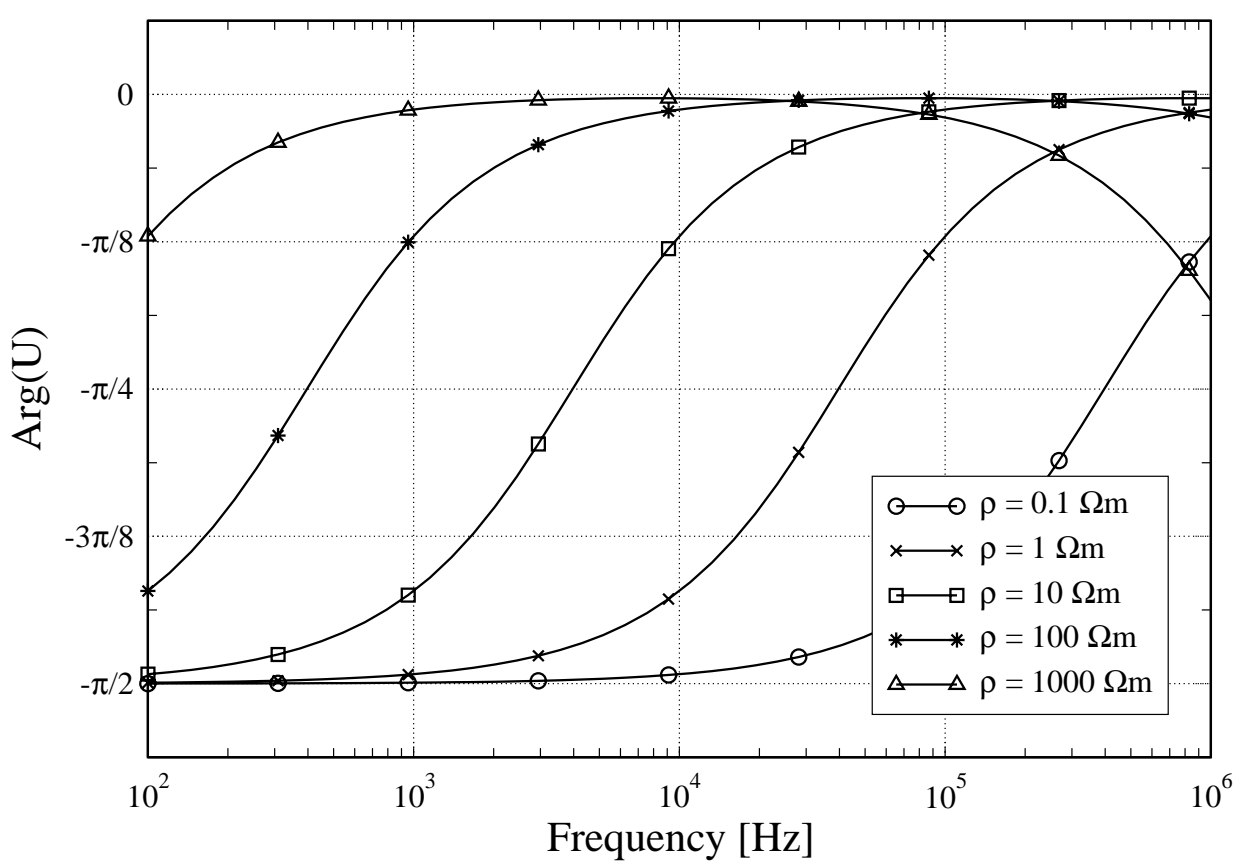

FIG. 9. Variation of the complex potential with frequency in magnitude (a) and phase (b) under quasi-static conditions for a unit square capacitive array ( $l=1 \mathrm{~m}, r=1 \mathrm{~m})$. Results for five values of resistivity are shown. Array elevation $h=1 \mathrm{~mm}$, relative permittivity $\varepsilon_{r}=10$, injected current $I=10 \mathrm{~mA}$. Magnitude values for an equivalent DC array are shown as dashed lines. 
(a) Magnitude

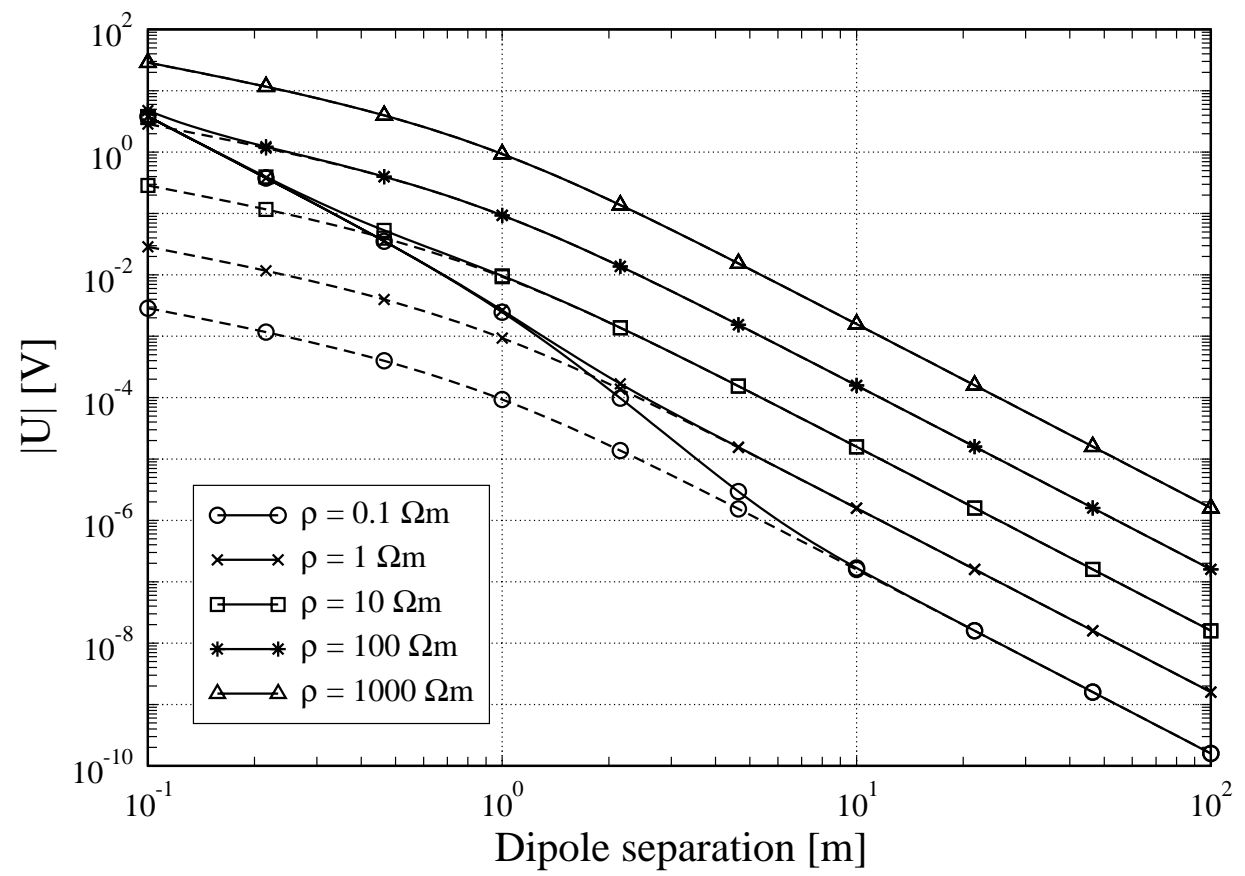

(b) Phase

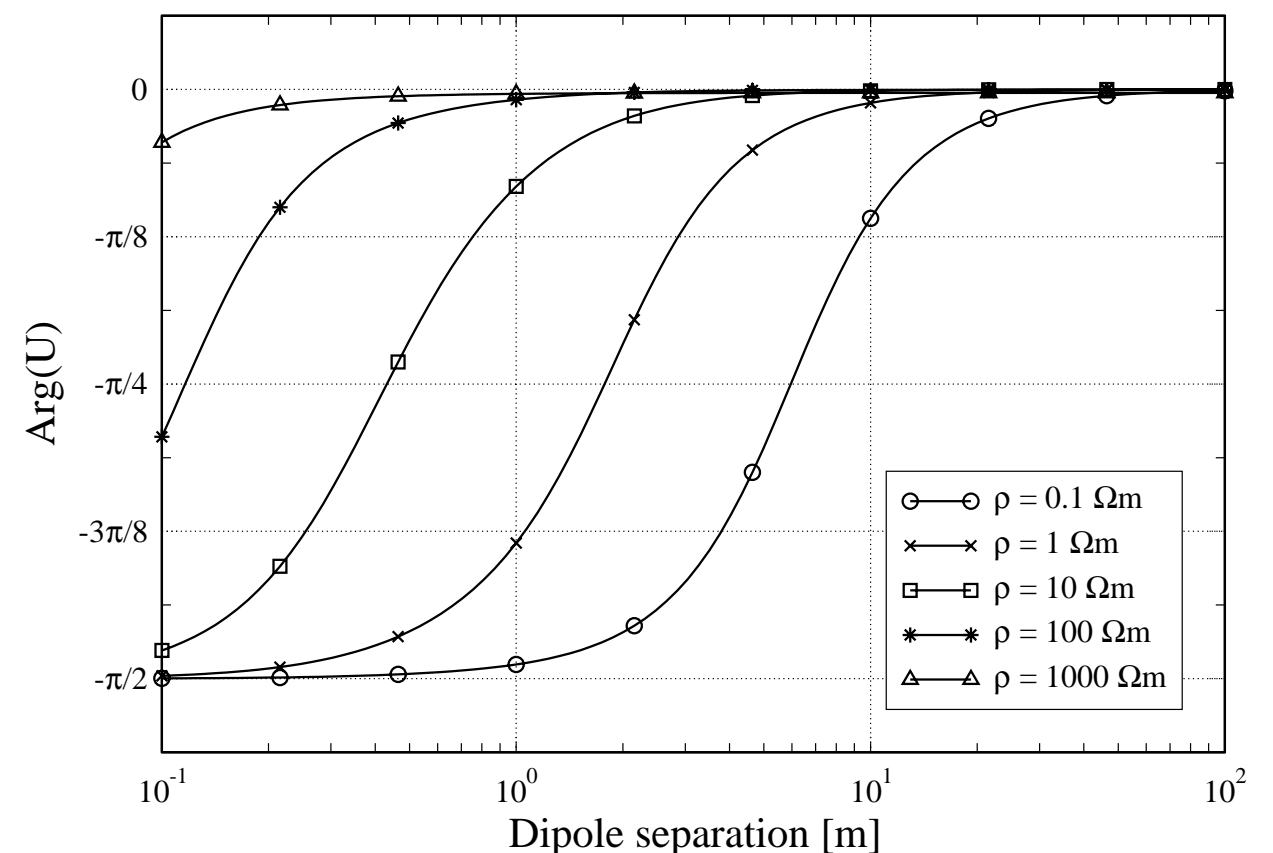

FIG. 10. Variation of the complex potential with dipole separation in magnitude (a) and phase (b) under quasi-static conditions for a capacitive array in equatorial configuration. Results for five values of resistivity are shown. Dipole length $l=1 \mathrm{~m}$, array elevation $h=1 \mathrm{~mm}, \varepsilon_{r}=10, f=15 \mathrm{kHz}, I=10 \mathrm{~mA}$. Magnitude values for an equivalent DC array are shown as dashed lines. 
(a) Magnitude

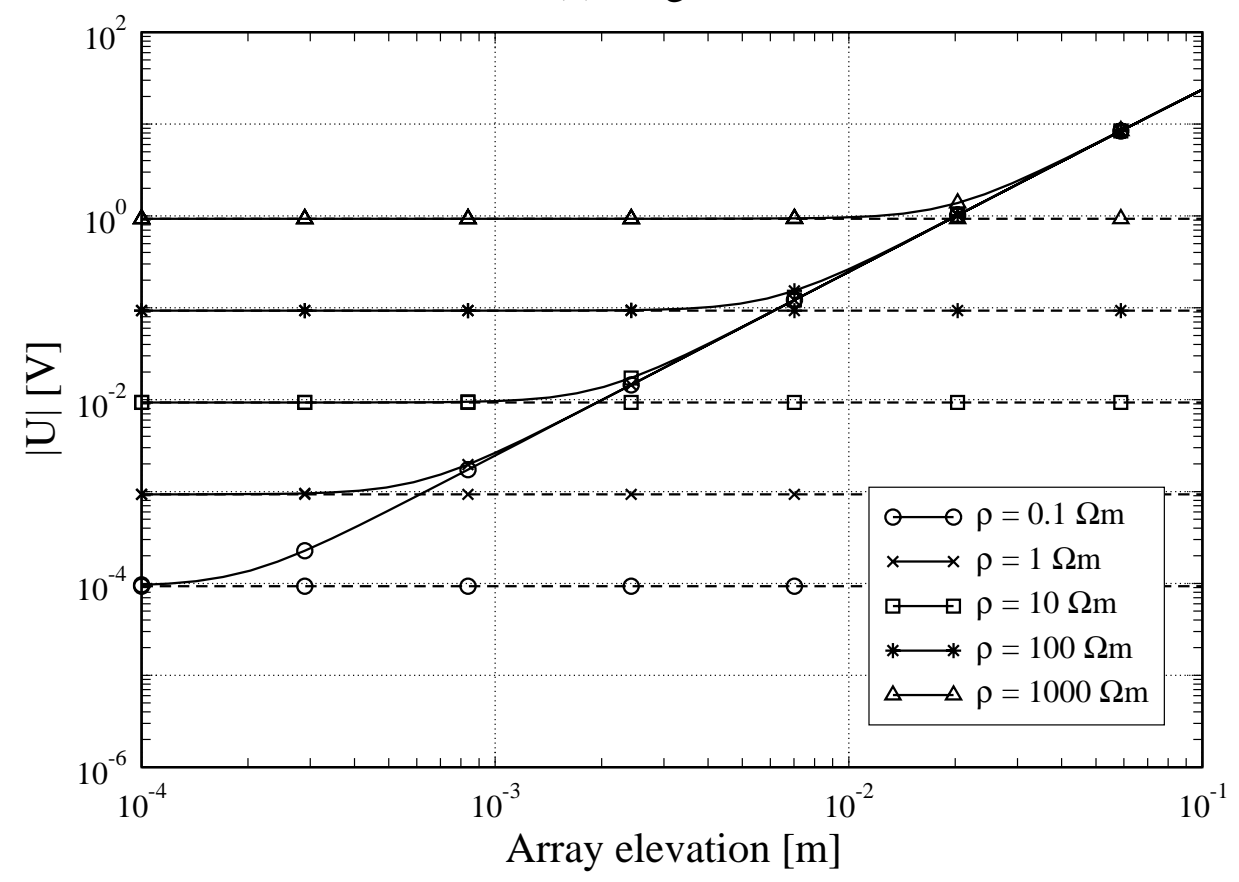

(b) Phase

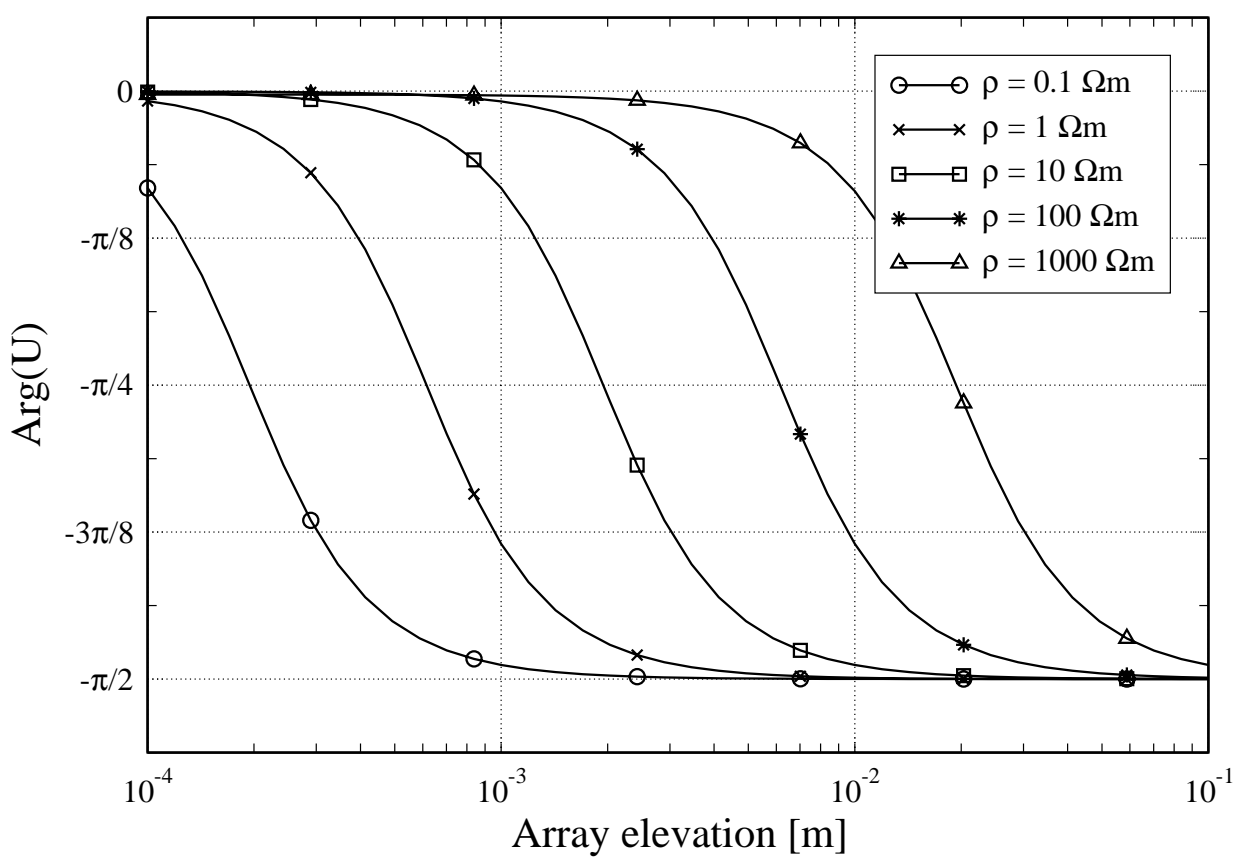

FIG. 11. Variation of the complex potential with array elevation in magnitude (a) and phase (b) under quasi-static conditions for a capacitive array in equatorial configuration. Results for five values of resistivity are shown. Dipole length $l=1 \mathrm{~m}$, dipole separation $r=1 \mathrm{~m}, \varepsilon_{r}=10, f=15 \mathrm{kHz}, I=10 \mathrm{~mA}$. Magnitude values for an equivalent DC array are shown as dashed lines. 
(a) Magnitude

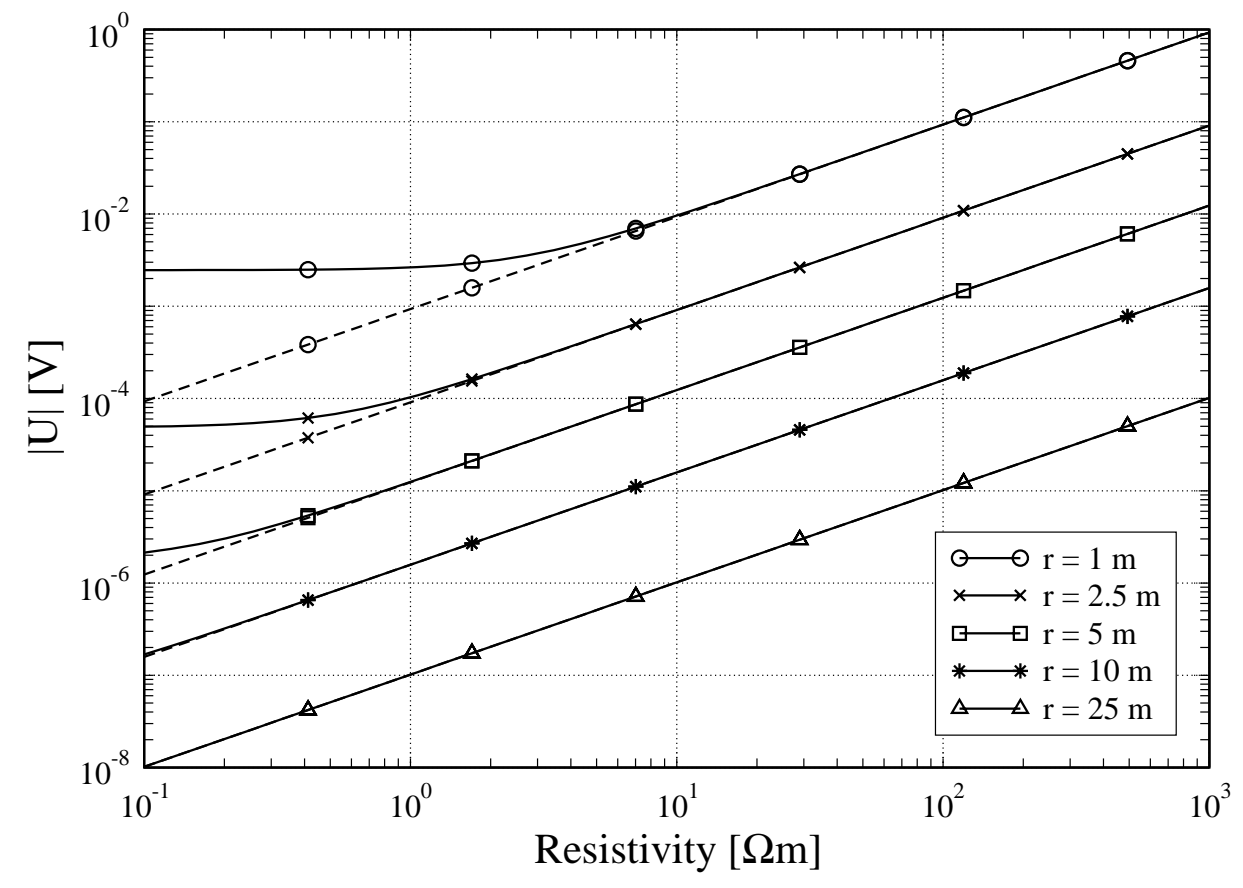

(b) Phase

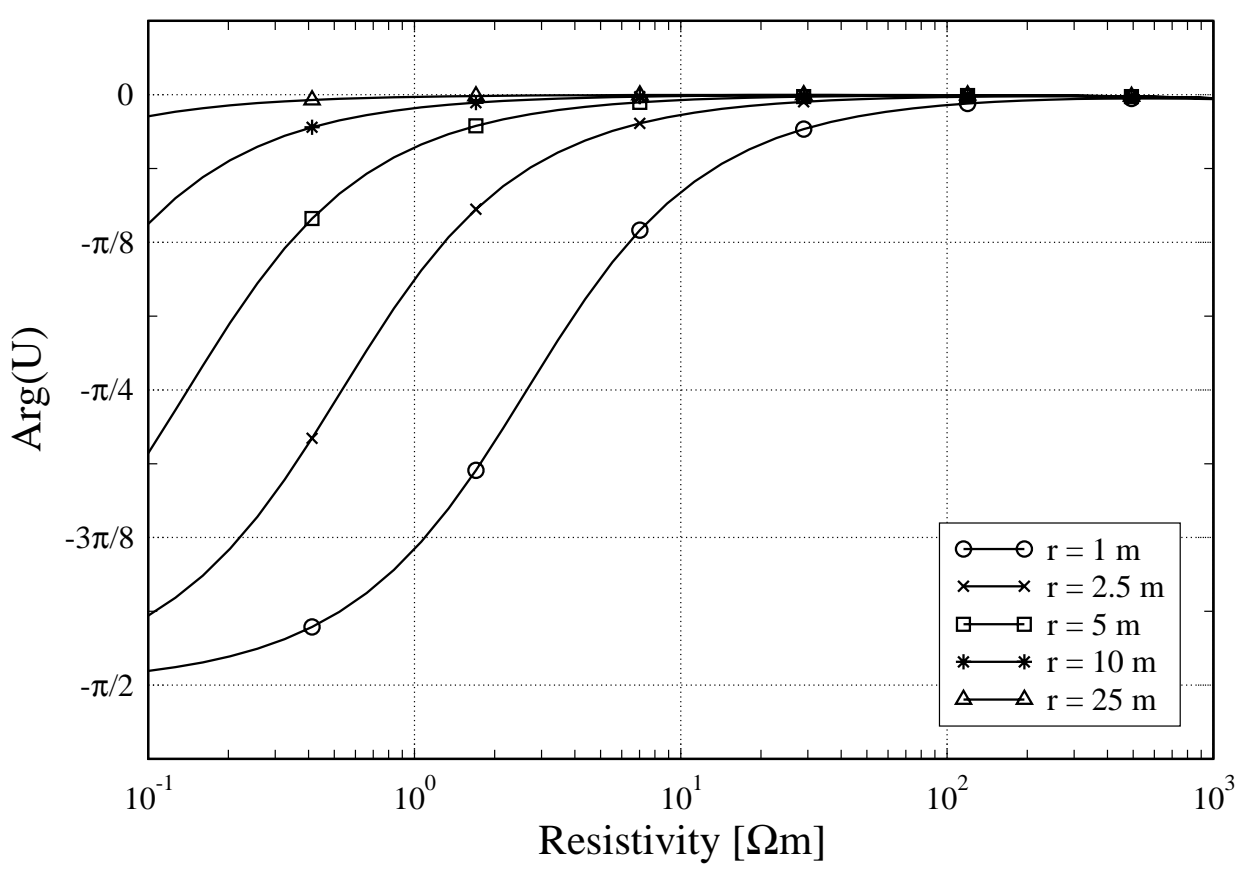

FIG. 12. Variation of the complex potential with resistivity in magnitude (a) and phase (b) under quasi-static conditions for a capacitive array in equatorial configuration. Results for five values of the dipole separation $r$ are shown. Dipole length $l=1 \mathrm{~m}$, array elevation $h=1 \mathrm{~mm}, \varepsilon_{r}=10, f=15 \mathrm{kHz}, I=10 \mathrm{~mA}$. Magnitude values for an equivalent DC array are shown as dashed lines. 
(a) Magnitude

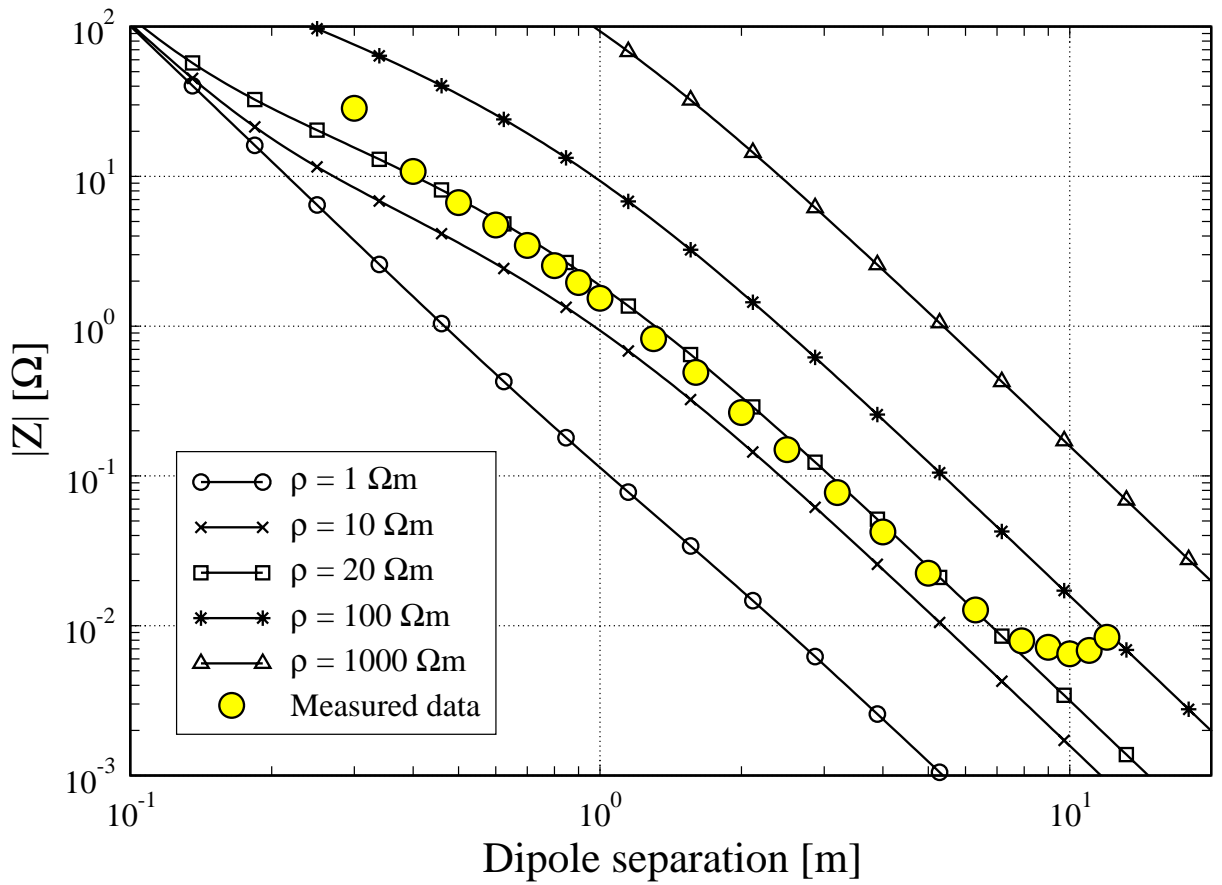

(b) Phase

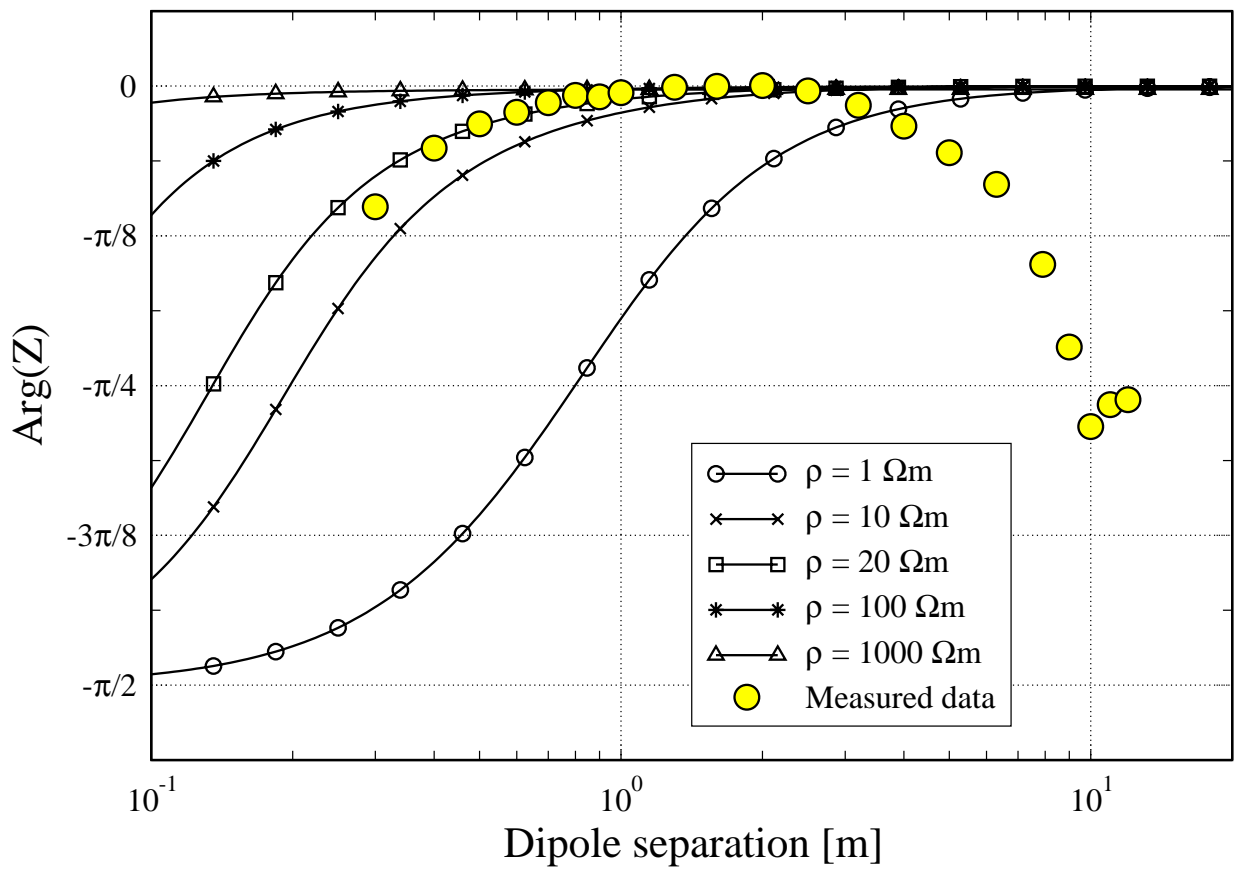

FIG. 13. Complex transfer impedances for the moveout survey in equatorial configuration: comparison of measured data with quasi-static model in magnitude (a) and phase (b). Results for five values of resistivity are shown; model array elevation remained fixed at $h=0.5 \mathrm{~mm}$. 
(a) Magnitude

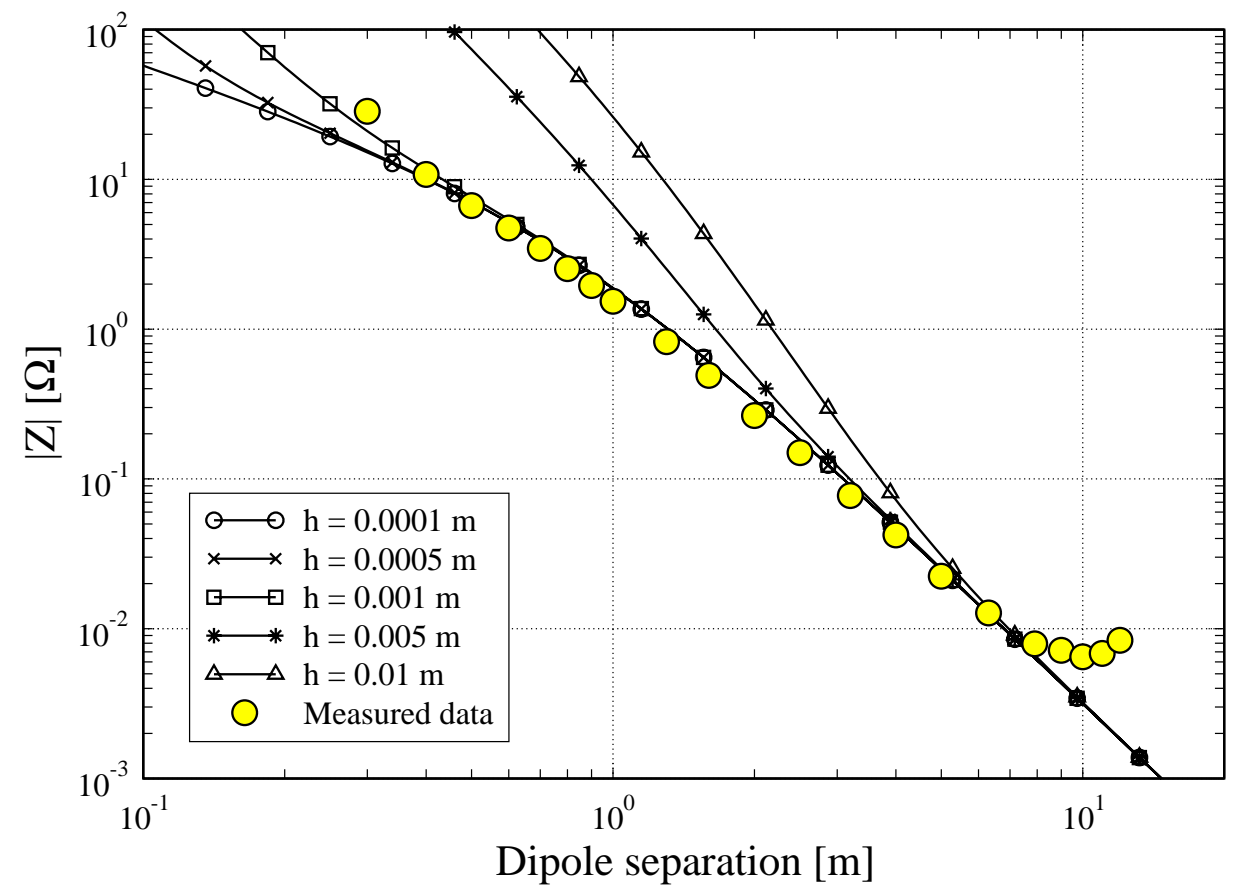

(b) Phase

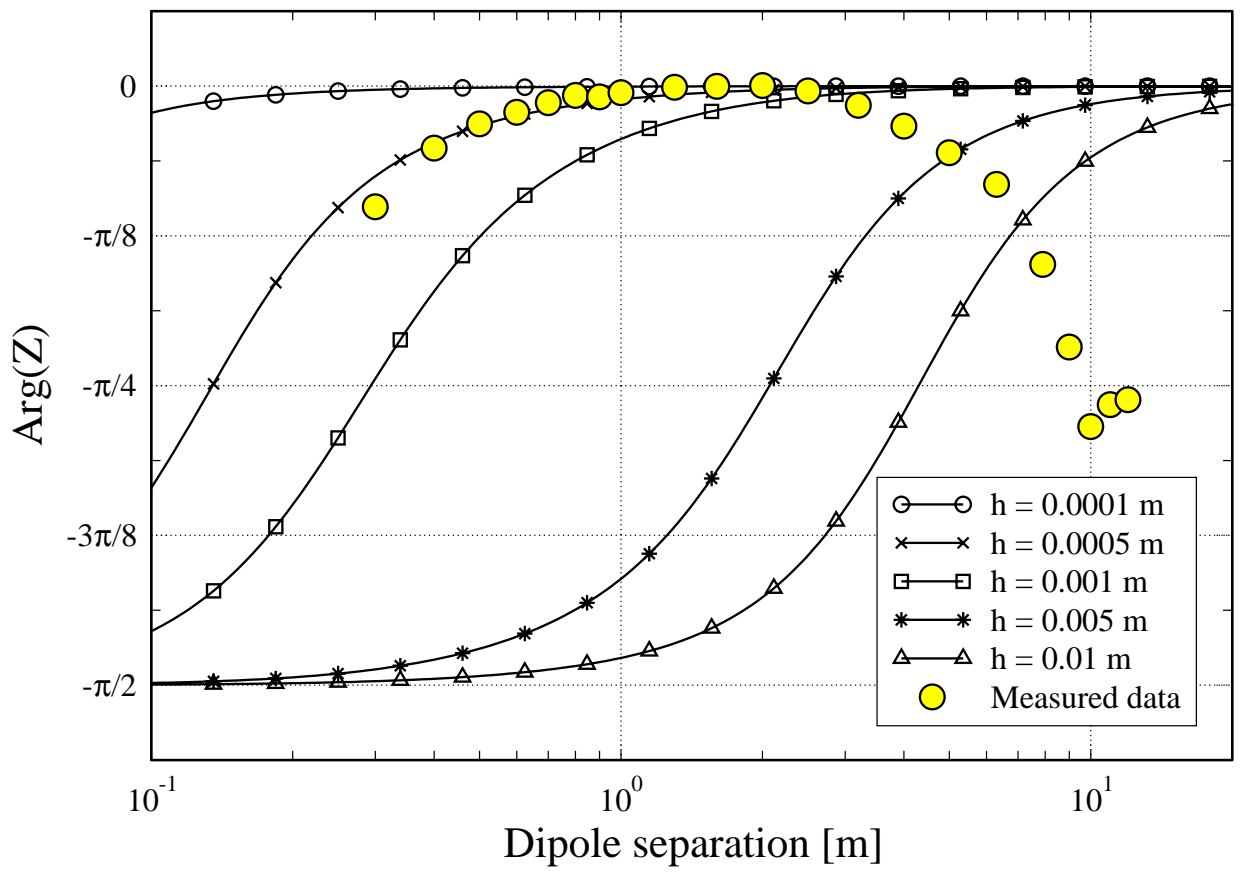

FIG. 14. Complex transfer impedances for the moveout survey in equatorial configuration: comparison of measured data with quasi-static model in magnitude (a) and phase (b). Results for five values of array elevation are shown; model resistivity remained fixed at $\rho=20 \Omega \mathrm{m}$. 


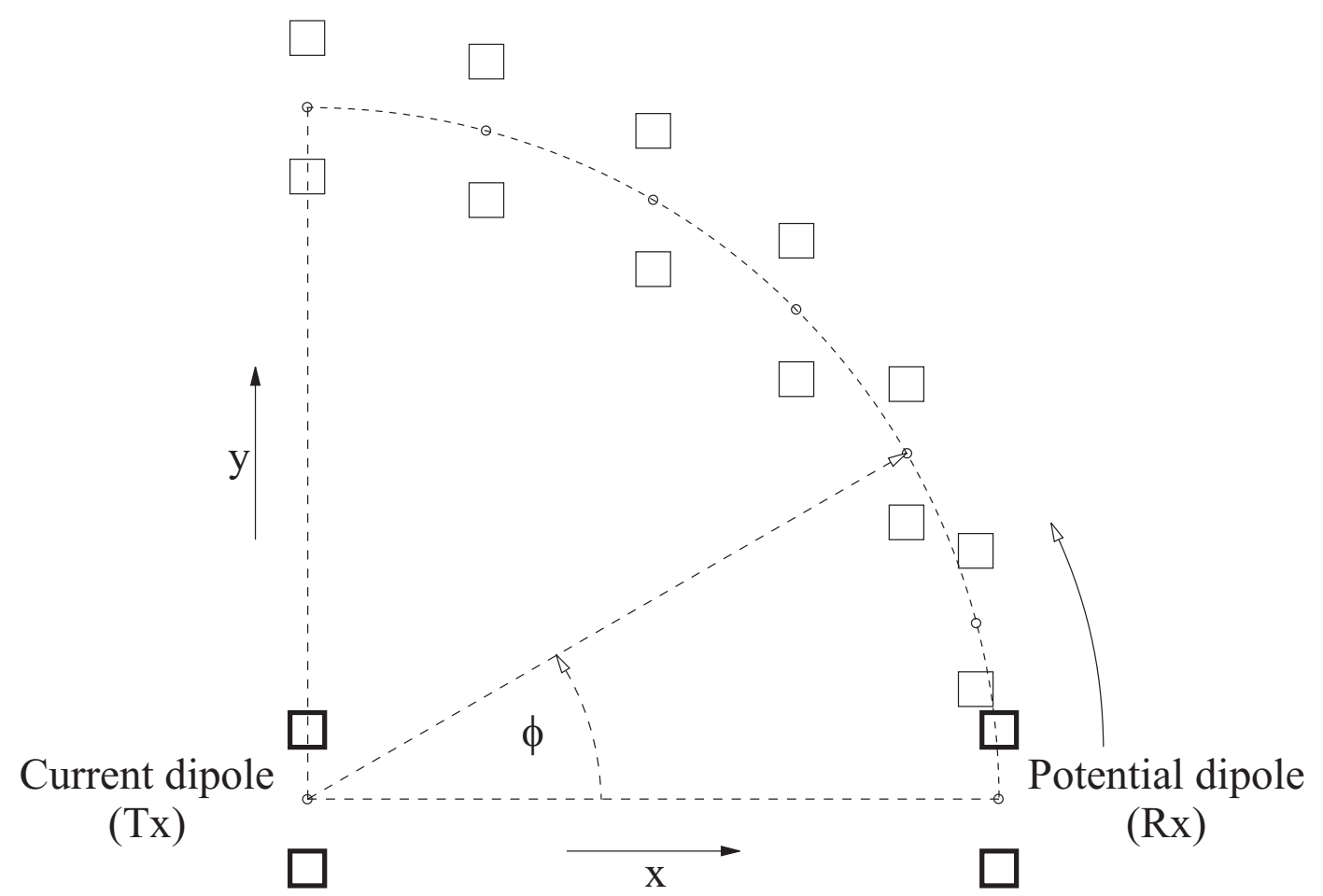

FIG. 15. Directional survey in parallel configuration to determine the azimuthal variation of the transfer impedance. 
(a) Magnitude

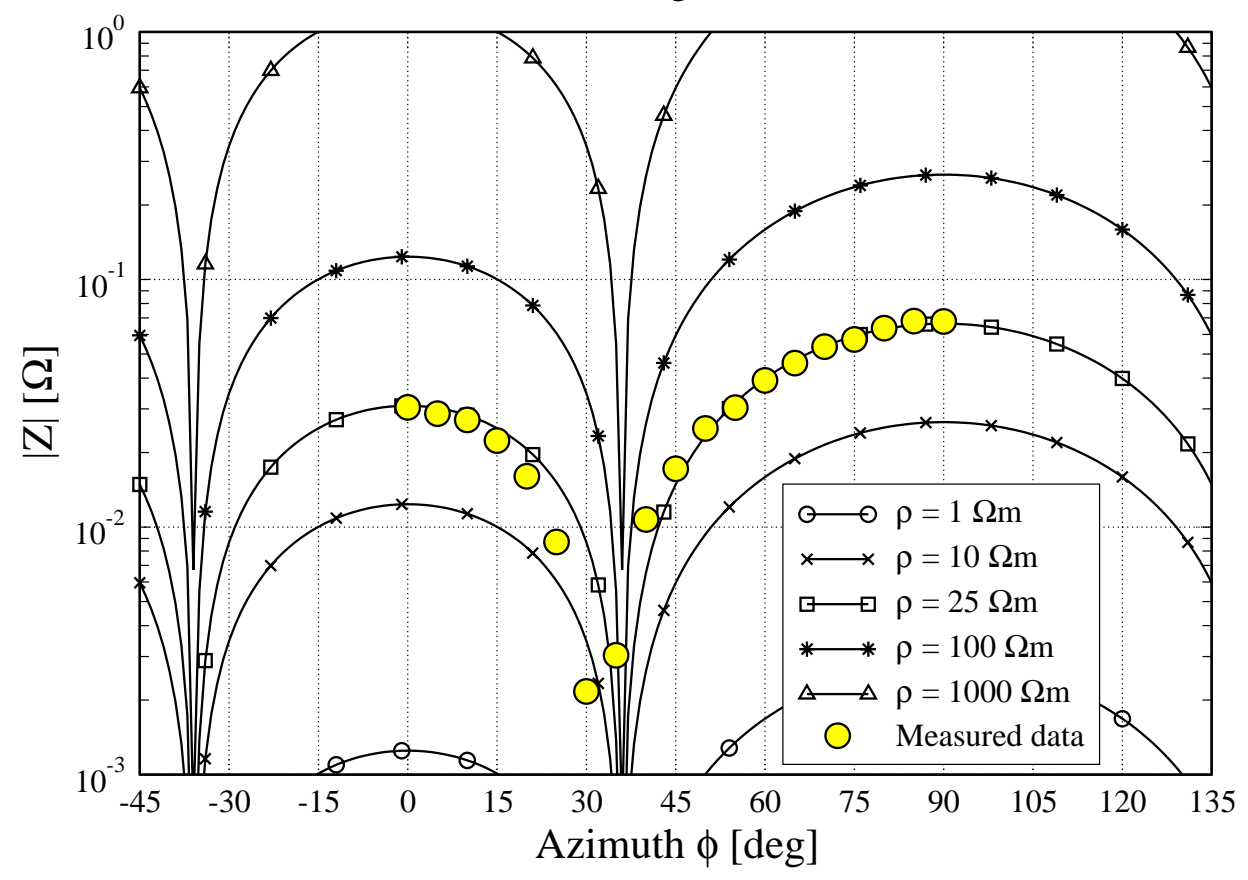

(b) Phase

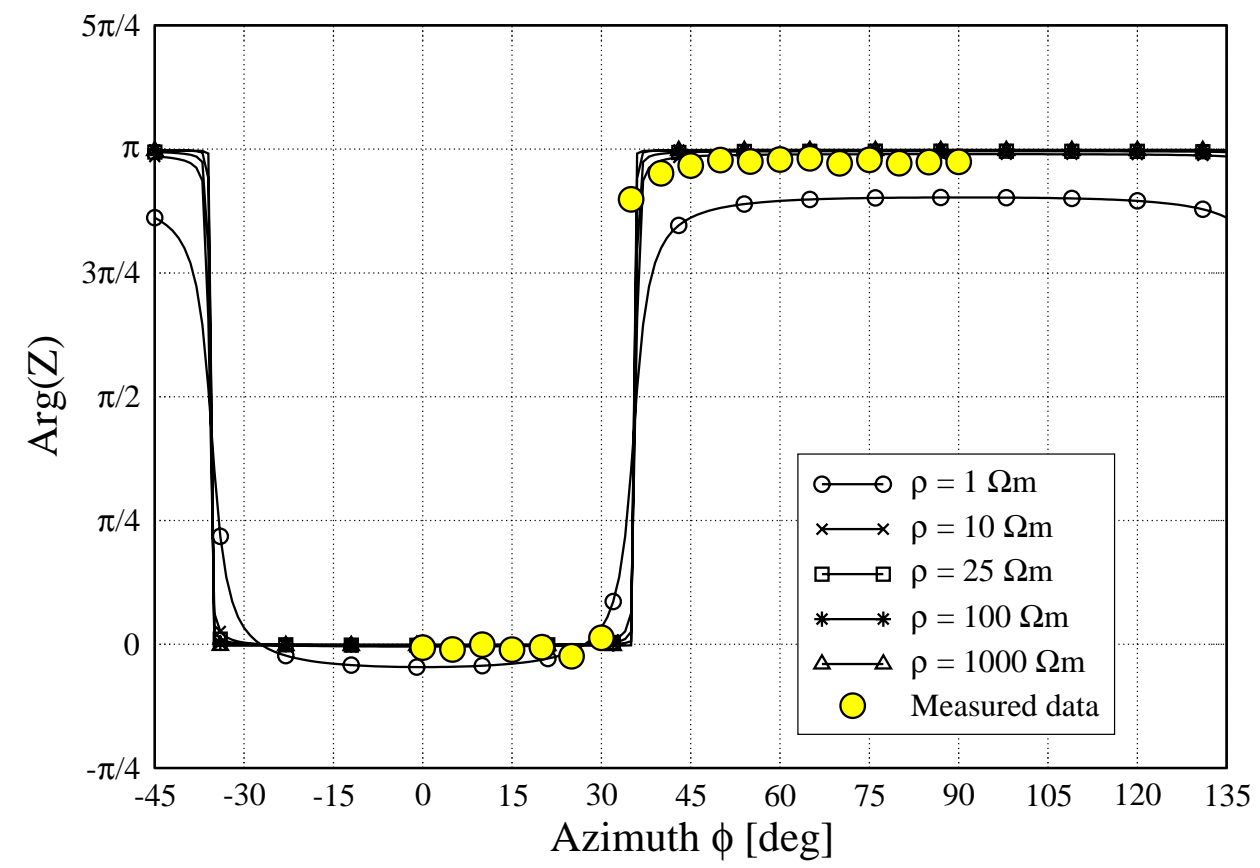

FIG. 16. Transfer impedances for the directional survey in parallel configuration: comparison of measured data with quasi-static model in magnitude (a) and phase (b). Results for five values of resistivity are shown; model array elevation remained fixed at $h=1 \mathrm{~mm}$. 
(a) Magnitude

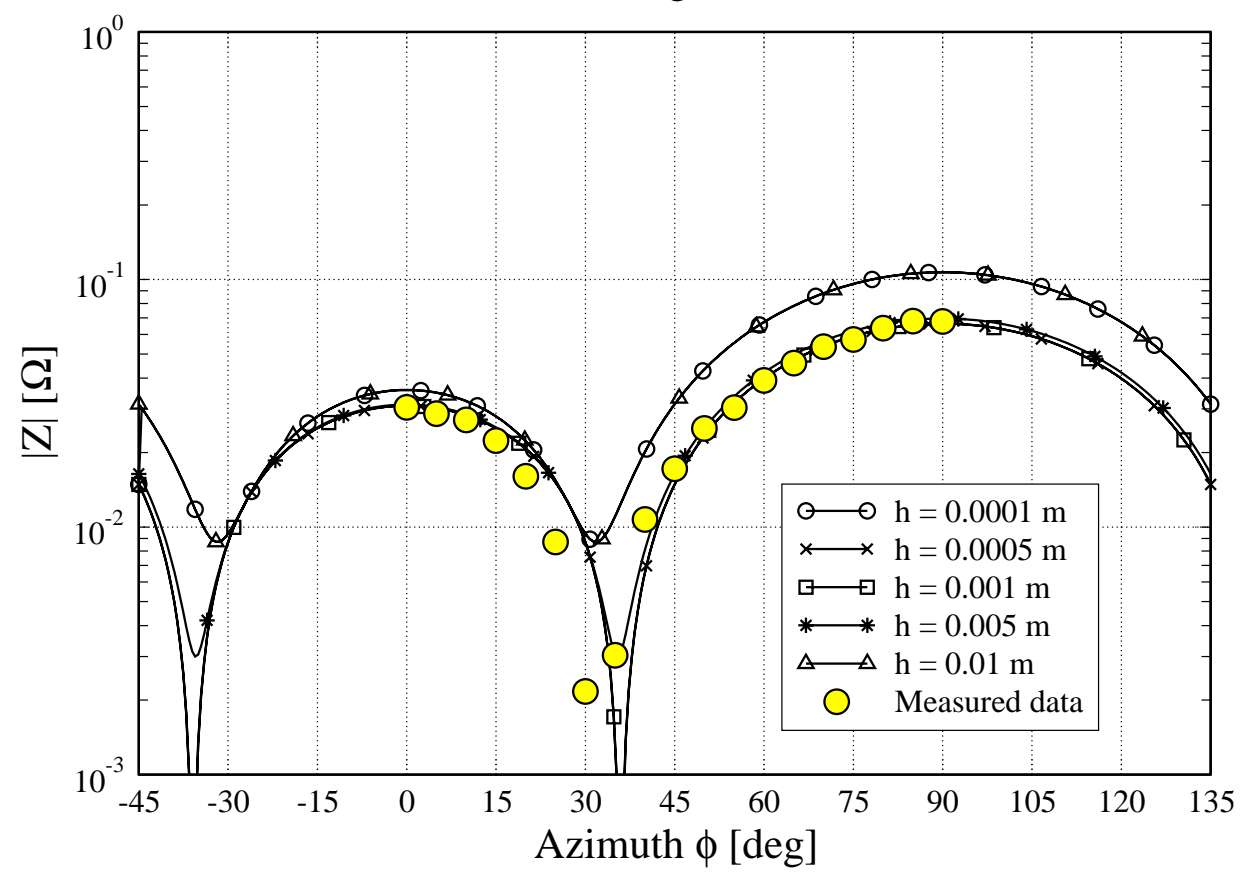

(b) Phase

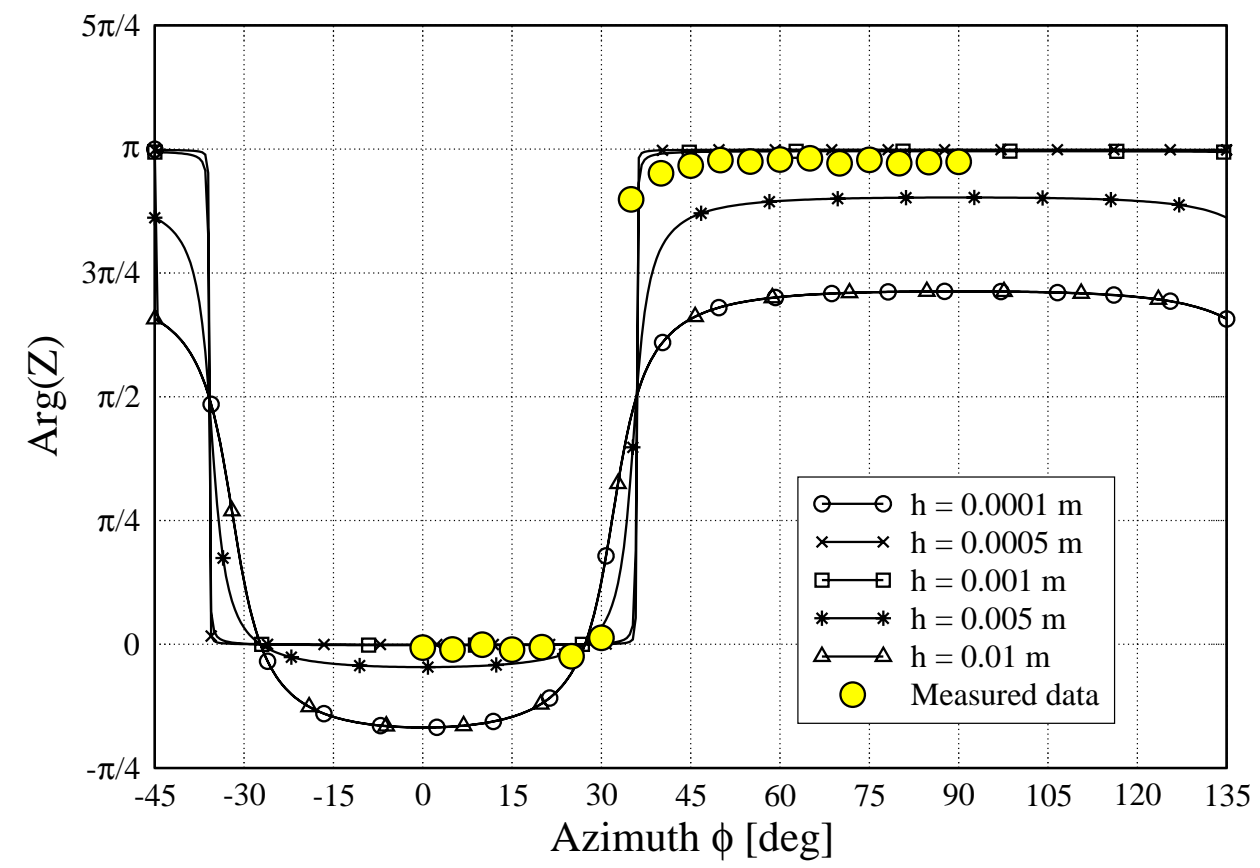

FIG. 17. Transfer impedances for the directional survey in parallel configuration: comparison of measured data with quasi-static model in magnitude (a) and phase (b). Results for five values of array elevation are shown; model resistivity remained fixed at $\rho=25 \Omega \mathrm{m}$. 